\title{
Design of General Kinematotropic Mechanisms
}

\author{
Qiang Zeng ${ }^{1}$, Kornel F. Ehmann, Jian Cao \\ Department of Mechanical Engineering, Northwestern University, \\ 2145 Sheridan Road, Evanston, IL 60208-3111, USA \\ ${ }^{1}$ Corresponding author. Tel.: 1-847-275-1208. E-mail address: qiangzengnu@gmail.com
}

\begin{abstract}
The general mobility of a linkage mechanism is determined during its continuous motions, but there is a class of mechanisms with kinematotropic properties whose mobility can be changed at singularities of their pose, while their kinematic structure remains unchanged in the different motion modes. This paper presents a systematic design method for general kinematotropic mechanisms. Firstly, the basic kinematic structures of spatial motions in serial and parallel forms are introduced. Secondly, the basic properties and design rules of kinematotropic mechanisms are defined, and the basic serial kinematic chains with variable constraint relations are presented. Thirdly, a systematic method for kinematic structural synthesis of general kinematotropic mechanisms is proposed. Finally, two types of motion transformations with four kinematotropic mechanisms are synthesized and analyzed as illustrative examples of the method.
\end{abstract}

Keywords: Kinematotropic mechanism; Mobility bifurcation; Parallel mechanism; Kinematic structural synthesis; Displacement group theory

\section{Introduction}

Kinematotropic mechanisms exhibit a very peculiar property in which a variation of the constraints can change their mobility. In other words, the output mobility can be changed between different motion modes when the structure is moving through a singularity pose of mobility bifurcation, and when the constraint relationships between axes or directions of different joints are variable in the same structure. Taking a single closed loop kinematotropic mechanism as an example, as shown in Fig. 1, the output motion of linkage (1) relative to linkage (2) is variable. When the axes of revolute joints $r_{1}$ and $r_{2}$ are collinear and the prismatic joints $t_{1}$ and $t_{2}$ are placed in the same direction, the mechanism is set into a state of mobility bifurcation. The output motion mode of linkage (1) relative to linkage (2) is a rotation $1 R$ when $r_{1}$ and $r_{2}$ are collinear and $t_{1}$ and $t_{2}$ are non-equidirectional, while the output motion mode of linkage (1) relative to linkage (2) is a translation $1 T$ when $r_{1}$ and $r_{2}$ are non-collinear and $t_{1}$ and $t_{2}$ are equidirectional. The output motion mode can be transformed between $1 R$ and $1 T$, because the constraint relationships are variable between axes or directions of different joints.

The kinematotropic property enhances the motion capabilities and allows spatial closed loop mechanisms to become self-reconfigurable. For kinematotropic mechanisms with multi-loop subchains, Wohlhart [1] and Roeschel [2] presented three multi-loop kinematotropic mechanisms and some kinematotropic linkages. Galletti and Giannotti [3] discussed principles of mobility of multi-loop kinematotropic mechanisms. Kinematotropic mechanisms with multiple motion modes can transform their mobility into more than two forms, e.g., Zlatanov et al. [4] presented a parallel mechanism that can generate four 3-DOFs (degrees of freedom) motion modes. Similarly, Galletti and Fanghella [5] presented a single-loop kinematotropic mechanism with three motion modes. 


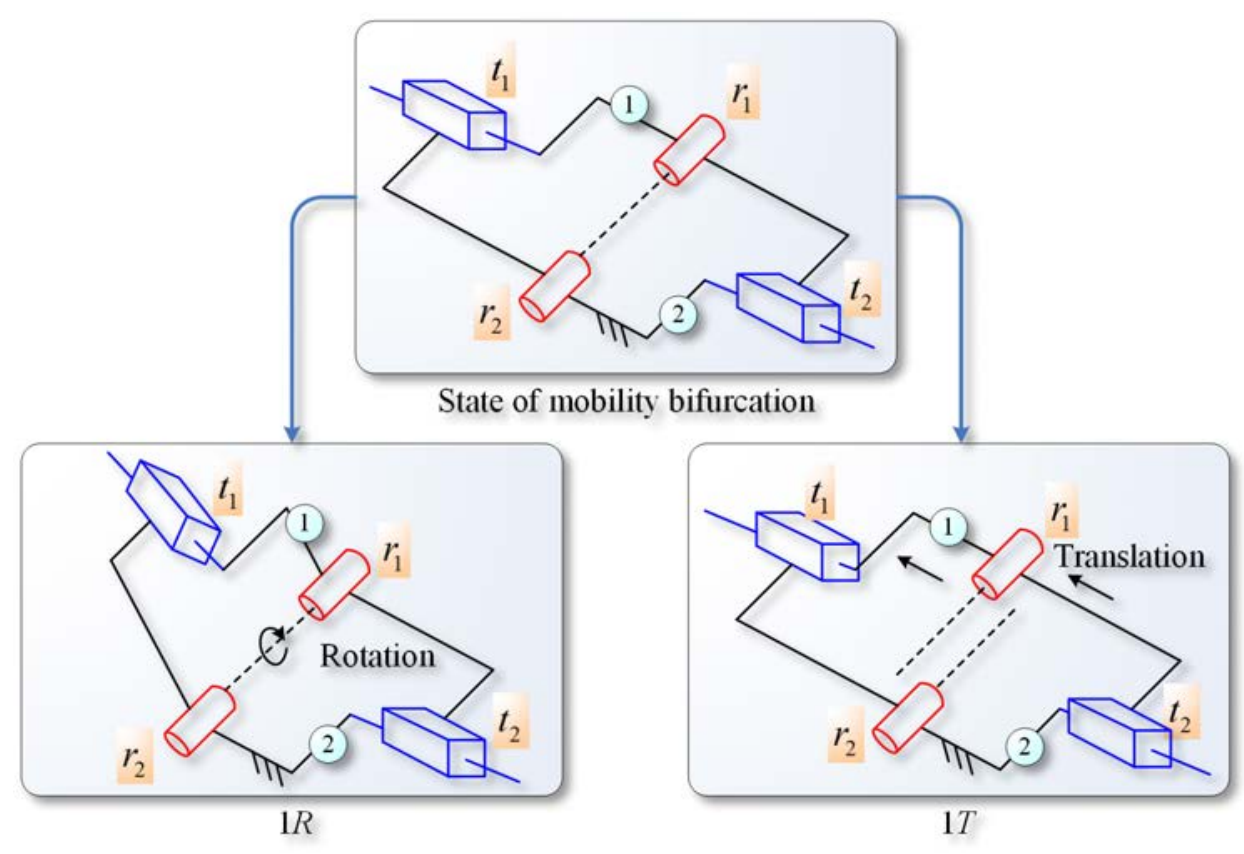

Fig. 1. Two motion modes of a single closed loop kinematotropic mechanism with $1 R \leftrightarrow 1 T$ form

As far as research on serial kinematic chains with variable constraint relations is concerned, Galletti and Fanghella [5, 6] presented a design method for kinematotropic mechanisms based on four basic kinematotropic single-loop chains. The chains can be modified to obtain different kinematotropic mechanisms, but their approach was limited to four basic chains. Actually, there exist kinematotropic mechanisms with other forms of serial kinematic chains with variable constraint relations as well. Thus, this paper will consider more basic serial kinematic chains with variable constraint relations and formulate basic principles for structural synthesis of general kinematotropic mechanisms.

In addition to the above works, many other design approaches for kinematotropic mechanisms have been developed and reported in the last decade. Among the more notable developments Fanghella et al. [7] used serial kinematic chains with variable constraint relations to synthesize some sub-6-DOFs parallel mechanisms with mobility bifurcation, while Refaat et al. [8] proposed a new parallel mechanism with motion bifurcation of the end-effector for machine tool applications. Kong et al. [9] presented a method for the type synthesis of parallel kinematotropic mechanisms with 3-DOFs spherical and translational motion modes. Li and Hervé [10] synthesized a special family of parallel mechanisms whose moving platform can undergo a bifurcation of Schoenflies motion. Gogu [11] presented a new family of maximally regular 2T1R-type parallel manipulators with bifurcated spatial motion of the moving platform along with solutions using uncoupled motions. Lee and Hervé [12-14], in turn, proposed the concept of discontinuous mobility mechanisms that can be regarded as kinematotropic linkages.

As far as the property of structures is concerned, Balli and Chand [15, 16] developed an analytical method for the determination of the motion and of the path generating mechanism with variable structure between two extreme positions. Yan and Kang [17] proposed a design methodology for the configuration synthesis of variable chains. The variable chains with variable structures can change output motions during the operation process, which have been developed in other researches [18-21]. In a related area of research, metamorphic [22-27] and reconfigurable [28-30] mechanisms have also received much attention in the last decade. 
In this paper, compared to the serial kinematic chains with variable constraint relations presented in the literature $[1,2,5,6]$, more basic serial kinematic chains with variable constraint relations will be developed, and the basic design principles for their structures and constraints will be formulated. Furthermore, other types of motion modes with different combinations of rotations and translations will be considered for the mobility transformation of kinematotropic mechanisms. The organization of this paper is as follows. In Section 2, the conventional structures of spatial motions in serial and parallel forms are summarized and introduced as basic elements for structural synthesis of general kinematotropic mechanisms. In Section 3, the basic characteristics and essence of kinematotropic mechanisms, constraints and basic serial kinematic chains with variable constraint relations, and design rules are developed for the structural synthesis of general kinematotropic mechanisms. The systematic logical steps for the design of general kinematotropic mechanisms are proposed in Section 4. To demonstrate the applicability of the proposed method, two types of motion transformations with four kinematotropic mechanisms are synthesized and analyzed in Section 5 followed by conclusions in Section 6.

\section{Conventional serial and parallel mechanisms of spatial motions}

In a Cartesian coordinate frame, there are the three rotations and three translations along the three orthogonal axes. The three orthogonal axes are determined as the motion axes of rotations and translations. Thus, the output motion of a mechanism's end-manipulator, namely the $x_{1}$ rotations and $x_{2}$ translations moving along $x_{3}$ motion axes can be described in the form:

$$
x_{1} R x_{2} T\left(x_{3} D\left(x_{1}+x_{2}\right) F\right), x_{1}=1,2,3, x_{2}=1,2,3, x_{3}=1,2,3 \text { and } x_{3} \geq \max \left(x_{1}, x_{2}\right)
$$

where $x_{1} R$ and $x_{2} T$ represent the number of rotations and translations respectively, $x_{3} D$ represents the number of motion axes of rotations and translations, and $\left(x_{1}+x_{2}\right) F$ represents the number of DOFs, i.e., the sum of the number of rotations and translations. The maximal $x_{3}$ is 3 since there are only three orthogonal axes in a Cartesian coordinate frame. For example, a planar joint has one rotation and two translations. Correspondingly, the output motion of the planar joint can be represented as $1 R 2 T(3 D 3 F)$.

Based on the rotations and translations of the Cartesian coordinate frame, the spatial motions include 19 types of combinatorial forms in the three orthogonal directions as shown in Table 1 . The 19 types of spatial motions can be generated by different serial or parallel mechanisms. Corresponding to the different spatial motions, the conventional serial and parallel mechanisms with only revolute and prismatic joints will be summarized in the following sections, which can be considered as basic elements for structural synthesis of general kinematotropic mechanisms.

Table 1. Basic spatial motions

\begin{tabular}{c|l|l|l}
\hline \multirow{2}{*}{ Rotations } & \multicolumn{3}{|c}{ Translations } \\
\cline { 2 - 3 } & $1 T(1 D 1 F)$ & $2 T(2 D 2 F)$ & $3 T(3 D 3 F)$ \\
\hline \multirow{2}{*}{$1 R(1 D 1 F)$} & $1 R 1 T(1 D 2 F)$ & $1 R 2 T(2 D 3 F)$ & \multirow{2}{*}{$1 R 3 T(3 D 4 F)$} \\
\cline { 2 - 3 } & $1 R 1 T(2 D 2 F)$ & $1 R 2 T(3 D 3 F)$ & \\
\hline \multirow{2}{*}{$2 R(2 D 2 F)$} & $2 R 1 T(2 D 3 F)$ & $2 R 2 T(2 D 4 F)$ & \multirow{2}{*}{$2 R 3 T(3 D 5 F)$} \\
\cline { 2 - 3 } & $2 R 1 T(3 D 3 F)$ & $2 R 2 T(3 D 4 F)$ & \\
\hline $3 R(3 D 3 F)$ & $3 R 1 T(3 D 4 F)$ & $3 R 2 T(3 D 5 F)$ & $3 R 3 T(3 D 6 F)$ \\
\hline \hline
\end{tabular}




\subsection{Basic chains in serial form}

In a Cartesian coordinate frame, by considering only the revolute and prismatic joints, there are 12 basic serial chains $S B_{*}$ that can be used to generate the basic rotational and translational motions. The remaining spatial motions can be generated by the different combinatorial forms of the basic serial chains $S B_{*}$ to built composite serial chains $S C_{*}$ (without parallelogram joints), as shown in Table 2. The combinatorial rules are based on the displacement group theory operations of the related basic serial chains $[31,32]$. For example, the composite serial chains $S C_{*}$ of the spatial motion $2 R 3 T(3 D 5 F)$ can be determined by the different combinations of the basic serial chains $S B_{*}$ in nine forms as:

(1) $S C_{2 R 3 T(1)}$ consists of $S B_{2 R(1)}$ and $S B_{3 T}$;

(2) $S C_{2 R 3 T(2)}$ consists of $S B_{2 R(1)}, S B_{1 R 1 T(1)}$ and $S B_{1 T}$;

(3) $S C_{2 R 3 T(3)}$ consists of $S B_{2 R(1)}, S B_{1 R 1 T(2)}$ and $S B_{1 T}$;

(4) $S C_{2 R 3 T(4)}$ consists of $S B_{2 R(2)}$ and $S B_{3 T}$;

(5) $S C_{2 R 3 T(5)}$ consists of $S B_{2 R(2)}, S B_{1 R 1 T(1)}$ and $S B_{1 T}$;

(6) $S C_{2 R 3 T(6)}$ consists of $S B_{2 R(2)}, S B_{1 R 1 T(2)}$ and $S B_{1 T}$;

(7) $S C_{2 R 3 T(7)}$ consists of $2 S B_{1 R 1 T(2)}$ and $S B_{1 T}$ with intersecting axes;

(8) $S C_{2 R 3 T(8)}$ consists of $2 S B_{1 R 1 T(2)}$ and $S B_{1 T}$ with orthogonal axes.

(9) $S C_{2 R 3 T(9)}$ consists of $S B_{1 R 1 T(2)}$ and $S B_{1 R 2 T}$ with orthogonal axes.

In a similar manner of the combinations, there are 74 forms of serial chains that can be summarized for the 19 types of spatial motions. In Table 2., the prismatic and revolute joints are marked by blue rectangles and red cylinders, respectively. The black heavy lines and dashed lines represent linkages and axes of revolute joints.

\subsection{Basic mechanisms in parallel form}

Based on the combinations of above 12 basic serial chains $S B_{*}$ and displacement group operations [31, 32], the related parallel mechanisms can be developed. There are 11 basic parallel chains $P B_{*}$ can be determined to generate the basic rotational and translational motions, as shown in Table 3 . The remaining spatial motions can be generated by the different combinatorial forms of the basic parallel chains $P B_{*}$ to built composite parallel chains designated as $P C_{*}$. For example, the determination of the composite parallel chain $P C_{2 R 1 T(3 D)}$ can be accomplished by the combination of the basic parallel chain $P B_{2 R}$ and $P B_{1 T(2)}$ to generate the output motion $2 R 1 T(3 D 3 F)$.

In analogy to the method of combination and displacement group operations [31, 32], there are 39 forms of parallel chains that can be summarized for the 19 types of spatial motions. This case is shown in Table 3. The output moving platforms of the parallel chains are marked by a black hatched part in the center of the structure. The end-linkage of each subchain is assumed to be fixed to the frame and marked by three black oblique lines, while the output motions are generated by the output moving platforms relative to the frame. 
Design of General Kinematotropic Mechanisms

Table 2. Basic serial kinematic chains

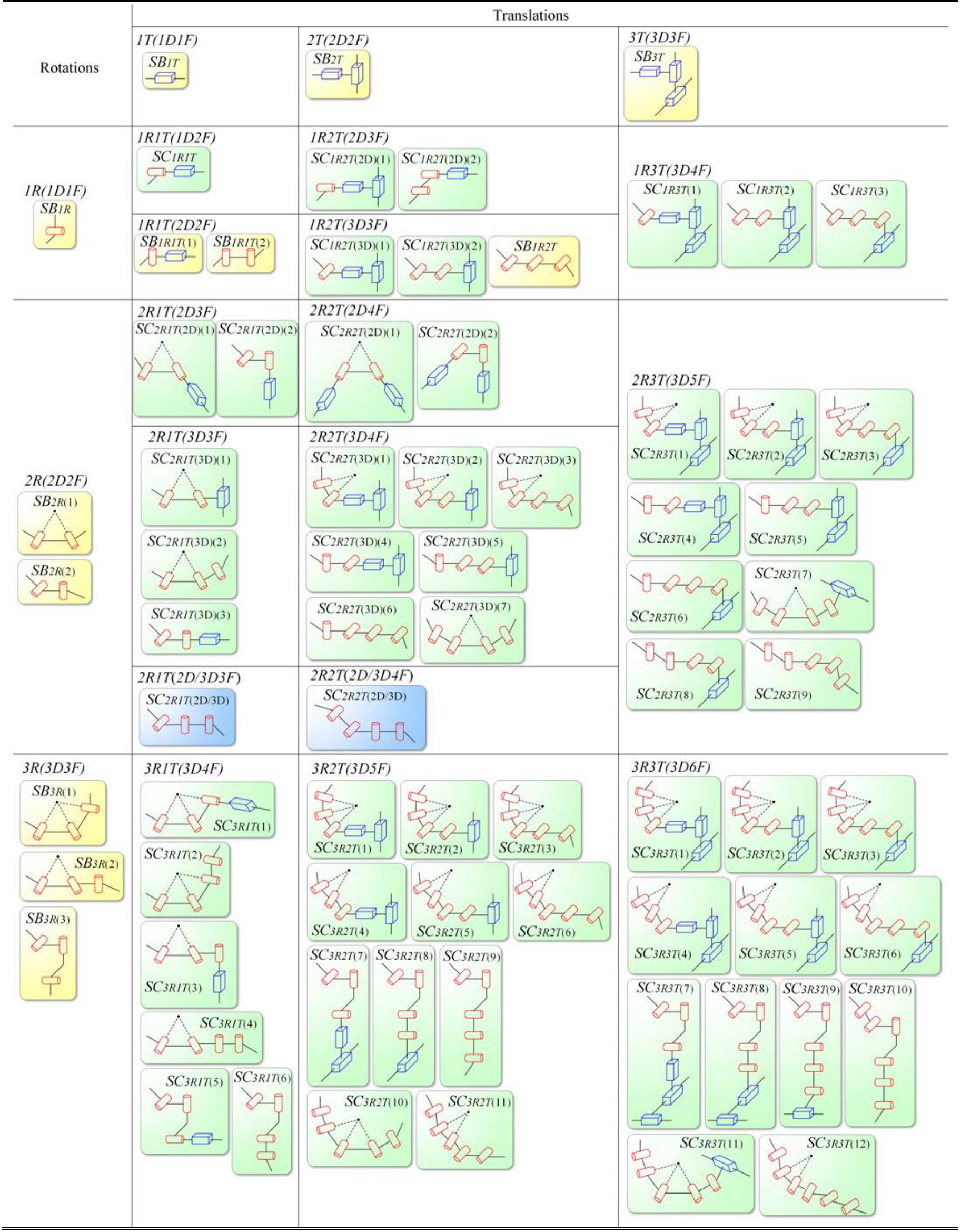


Design of General Kinematotropic Mechanisms

Table 3. Basic parallel mechanisms

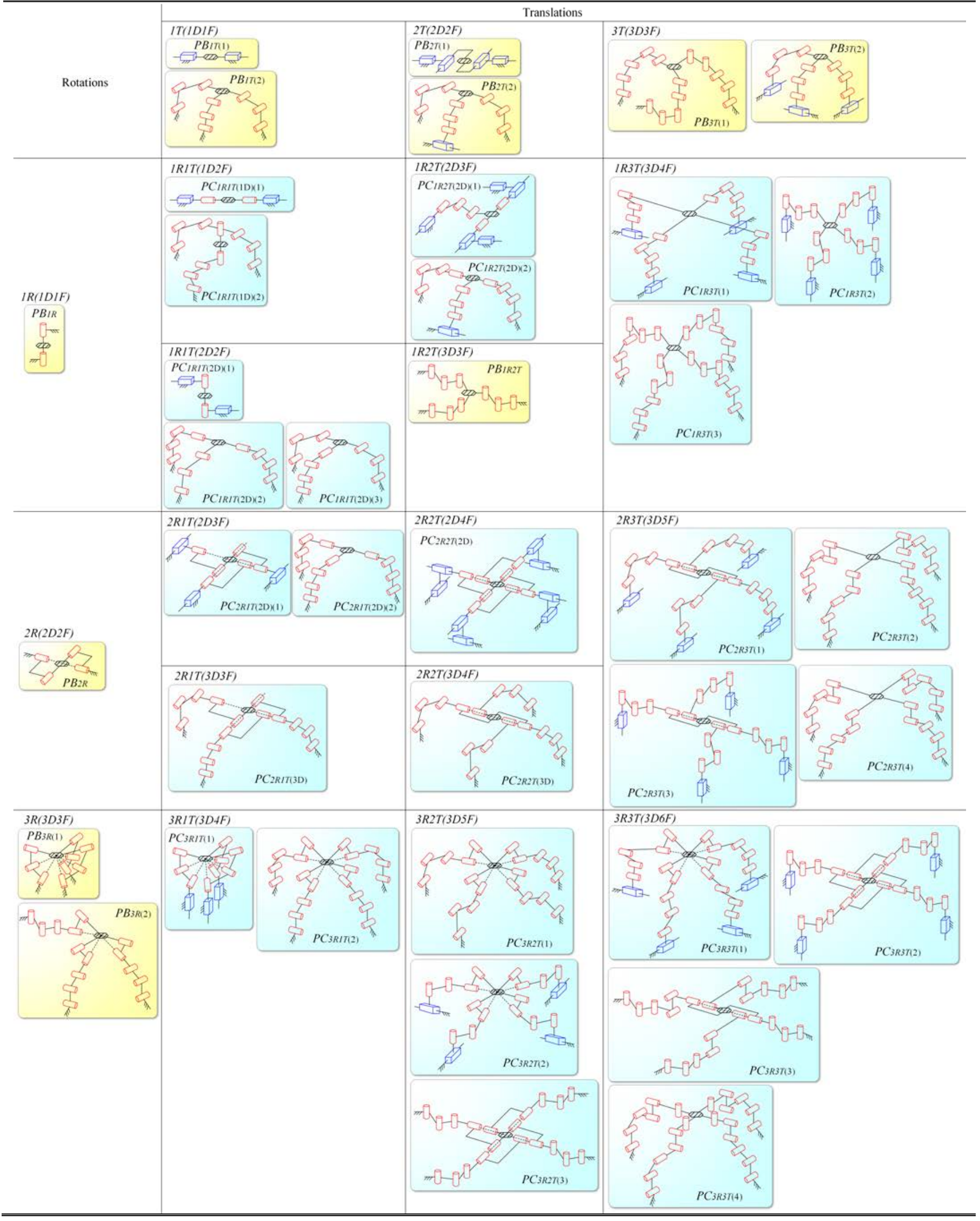




\section{Properties and design rules of kinematotropic mechanisms}

Kinematotropic linkage mechanisms are different from general linkage mechanisms due to their property of variable mobility. In order to design more general kinematotropic mechanisms, the properties of kinematotropic mechanisms such as the characteristics, essence, constraints and basic serial kinematic chains with variable constraint relations, and design rules should be determined and defined prior to the structural design.

\subsection{Characteristics of kinematotropic mechanisms}

The characteristics of kinematotropic mechanisms include the basic motion elements, structural forms, types of motion transformations, functional objects, characteristic structure and types of constraint forms between subchains. These can be formulated and clarified as follows.

\subsubsection{Basic motion elements}

In a fixed reference frame $O-x y z$, the basic motion elements of kinematotropic mechanisms can be determined as rotation $R$, translation $T$, and planar motion $G$. The rotation and translation are displacement subsets of the planar motion. The three motions can be used to synthesize kinematotropic mechanisms. The design and motion description of kinematotropic mechanisms can be developed based on the three basic motion elements. Other spatial motions, such as spherical, helical, conical, etc. motions are not included in this design method.

\subsubsection{Structural forms}

The basic structural forms of kinematotropic mechanisms can be categorized into four types, namely:

(1) the serial form with a serial subchain,

(2) the parallel form with a single closed loop and dual subchains,

(3) the parallel form with multi-loop and multiple subchains, and

(4) the hybrid form with multi-loops and multiple moving platforms.

In the hybrid form, the moving platform can be defined as a linkage that connects more than two subchains. The structural form should be determined before the structural synthesis of a kinematotropic mechanism is undertaken.

\subsubsection{Types of motion transformations}

As far as the transformation property of kinematotropic mechanisms is concerned, the types of motion transformations can be classified into four types as:

(1) the transformation of the number of DOFs $F$,

(2) the transformation of the type of mobility between rotation $R$ and translation $T$,

(3) the transformation of the number of motion axes $D$, and

(4) the transformation of the motions along the different motion axes.

The type of motion transformation of a kinematotropic mechanism can consist of a single type of motion transformation or a multiple types of motion transformations chosen from the above four types. 


\subsubsection{Functional objects}

In a serial kinematic chain with variable constraint relations, the output motions can be changed by the motions of related joints. Hence, another important concept associated with kinematotropic mechanisms is related to the definition of the functional objects of the kinematotropic mechanism. Functional objects are joints that can change the constraint relations and affect the output motions of the mechanisms in its continuous motions. There are two types of functional object as:

(1) The constraint relations between axes or directions and combinatorial output motions of a mechanism are transformed by the related joints. These joints can be referred to as "domination" namely Dom . Dom -s are actuated joints, generally to transform the motion mode of the kinematotropic mechanism.

(2) Another functional object is the joint in which constraint relations between axes or directions and combinatorial output motions are transformed by a Dom. This will be referred to as "passiveness" namely Pas. The constraint relations and combinatorial output motions of a Pas are variable in the different motion modes of the kinematotropic mechanism.

Thus, all joints in a serial kinematic chain with variable constraint relations can be categorized into Dom and Pas. The connection between a Dom and a Pas can be applied by a linkage or a moving platform.

\subsubsection{Characteristic structure}

A kinematic chain will be considered as a serial kinematic chains with variable constraint relations when it includes a Dom and a Pas with variable constraints and combinatorial output motions. Thus, the basic characteristic structure of a serial kinematic chain with variable constraint relations can be expressed schematically as in Fig. 2., where the element $\operatorname{Dom}\left(n_{i}\right)$ connects $\operatorname{Pas}\left(n_{i-1}\right)$ and $\operatorname{Pas}\left(n_{i+1}\right)$ with the linkage or moving platform. The type of connections has the properties of variable constraints. The connections of $\operatorname{Pas}\left(n_{i-1}\right)$ and $\operatorname{Pas}\left(n_{i+1}\right)$ will generate the variable combinatorial output motions according to the motions of $\operatorname{Dom}\left(n_{i}\right)$.

For example, in Fig. 1., the constraint relations of the directions between prismatic joints $t_{1}$ and $t_{2}$ can be changed by the revolute joints $r_{1}$ and $r_{2}$ when the single loop chain is transformed as the motion mode $1 R$. Thus, the prismatic joints $t_{1}$ and $t_{2}$ are Pas -es, while the revolute joints $r_{1}$ and $r_{2}$ are Dom -s. Actually, the role of Dom-s and Pas-es in a kinematotropic mechanism can be also exchanged between each other by the variable constraint relations in the different motion modes. In Fig. 1., the prismatic joints $t_{1}, t_{2}$ will become Dom -s, while the revolute joints $r_{1}, r_{2}$ are Pas -es when the single loop chain is transformed as the motion mode $1 T$.

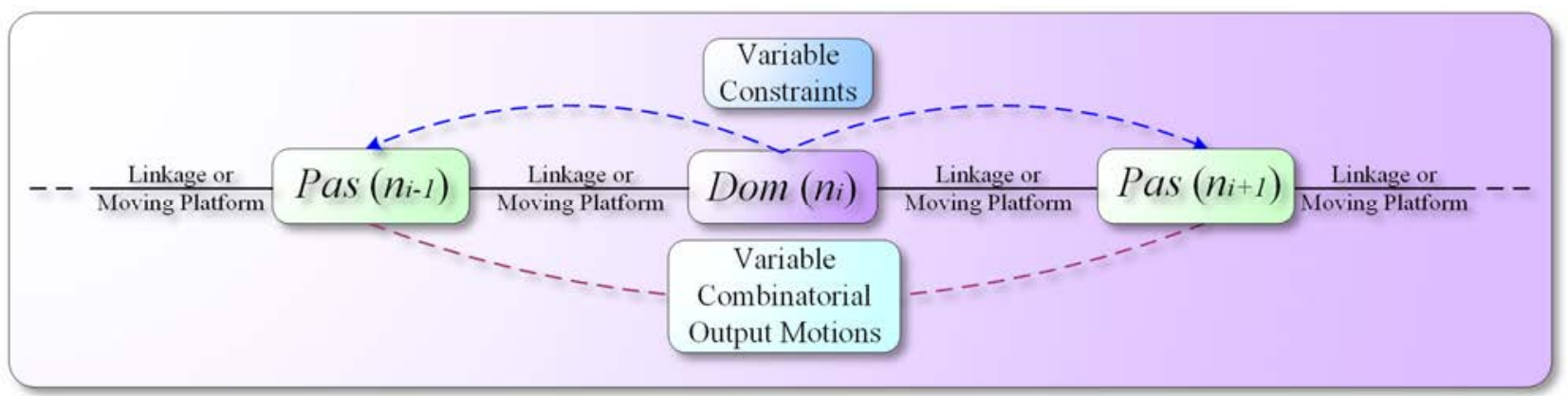

Fig. 2. Basic characteristic structure of a serial kinematic chain with variable constraint relations 


\subsubsection{Types of constraint forms}

In a parallel structure, a Dom can not only change the constraint relations of Pas -es in a subchain, but it can also change the constraint relations of Pas -es between the different subchains, which may be applied for all of subchains. Thus, in the light of the influence range of a Dom, there are two types of constraint forms for kinematotropic mechanisms with multiple subchains that can be defined, namely the self-transforming and inter-transforming forms as shown in Fig. 3. In the self-transforming form, the motions of Dom-s and Pas-es in a subchain only transform the variable constraints and combinatorial output motions of the corresponding subchain. In the inter-transforming form, the Dom-s and Pas-es are placed on different subchains and separated by a moving platform. The variable constraints and combinatorial output motions of the Pas-es are transformed by the different Dom-s between the different subchains.

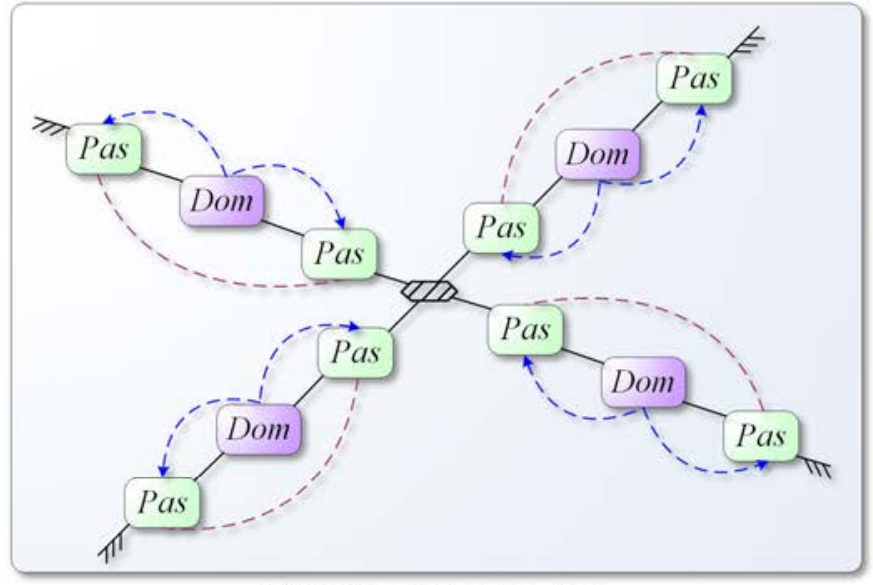

(a) Self-transforming form

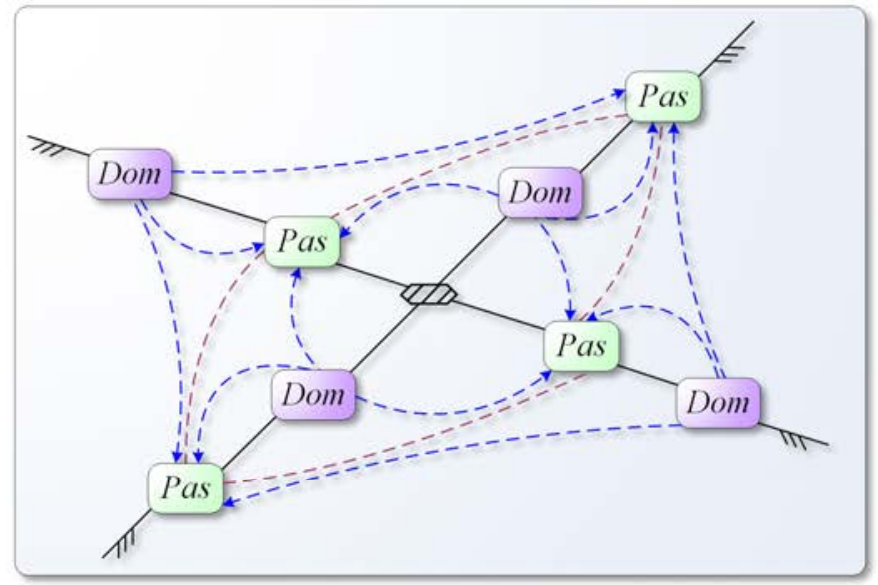

(b) Inter-transforming form

Fig. 3. Basic constraint forms of kinematotropic mechanisms

\subsection{Essence of kinematotropic mechanisms}

In accordance with the above characteristics, the essence of kinematotropic mechanisms can be stated as follows:

Essence of kinematotropic mechanisms: the continuous motions of Dom-s can transform the spatial constraint relations of an axis or direction $n_{i-1}$ and $n_{i+1}$ of two Pas-es, in other words, the combinatorial constraint structures are transformed. Thereby, the combinatorial output motions of two Pas-es are transformed under different constraint conditions to achieve the objective of mobility transformation of the kinematotropic mechanism.

\subsection{Constraints and basic serial kinematic chains with variable constraint relations}

\subsubsection{Basic constraint relations}

As far as the constraint relations between axes or directions of joints in kinematotropic mechanisms are concerned, there are three basic types of constraint relations that can be applied according to the operations of displacement group theory $[31,32]$, namely:

(1) $C_{a}$ : the axes of joints are collinear,

(2) $C_{d}$ : the axes or directions of joints are equidirectional, and 
(3) $C_{c}$ : the axes of joints are intersecting at a point.

The motion of a rotation can be described by as a rotation around a rotational axis, while a translation can only be represented by a motion along a direction, and the planar motion is featured by the normal of the plane which is determined as a direction of the normal. The constraints $C_{a}$ and $C_{c}$ are featured by the related axes, while the $C_{d}$ is determined by directions. Thus, the $C_{a}$ and $C_{c}$ are only related to the transformation of rotations, while the $C_{d}$ is related to the transformations of rotations, translations and planar motions. The direction of a revolute joint is determined as the direction of that is parallel to the rotational axis of the revolute joint. This is used to describe the constraint relations between rotational axis of revolute joint and moving direction of prismatic joint and planar motion.

\subsubsection{Basic serial kinematic chains with variable constraint relations}

In accordance with the basic motion elements $R, T$ and $G$ of kinematotropic mechanisms, there are three types of Dom-s that can be defined, namely:

(1) rotational $\operatorname{Dom}(R)$,

(2) translational $\operatorname{Dom}(T)$, and

(3) planar motion $\operatorname{Dom}(G)$.

The $\operatorname{Dom}(R)$ and $\operatorname{Dom}(G)$ can transform $C_{a}, C_{d}$ and $C_{c}$ constraints, while $\operatorname{Dom}(T)$ can only transform $C_{a}$ and $C_{c}$ constraints. $\operatorname{Dom}(R)$ and $\operatorname{Dom}(T)$ are subsets of $\operatorname{Dom}(G)$. Similarly, there exist six types of Pas-es that can also be defined, namely:

(1) $\operatorname{Pas}(R-R)$,

(2) $\operatorname{Pas}(T-T)$,

(3) $\operatorname{Pas}(G-G)$,

(4) $\operatorname{Pas}(R-T)$,

(5) $\operatorname{Pas}(R-G)$, and

(6) $\operatorname{Pas}(T-G)$.

The $C_{a}, C_{d}$ and $C_{c}$ constraints can be transformed in $\operatorname{Pas}(R-R)$, while the only $C_{d}$ constraint can be transformed in the other five types of Pas-es.

By considering the basic variable constraint relations and the different types of functional objects, the basic serial kinematic chains with variable constraint relations $K_{*}$ can be built by the related constraint combinations of Dom-s and Pas-es, as shown in Table 4. In the process of combination, a detachment point of a chain is defined as a point on a linkage to divide the linkage into two parts. The detachment points can be determined between the adjacent joints of the chosen constraint relations to provide the required motion transformations. Two Pas-es corresponding to the different constraint relations can be combined at the detachment point by a Dom to form a serial kinematic chain with variable constraint relations. 
Design of General Kinematotropic Mechanisms

Table 4. Basic serial kinematic chains with variable constraint relations

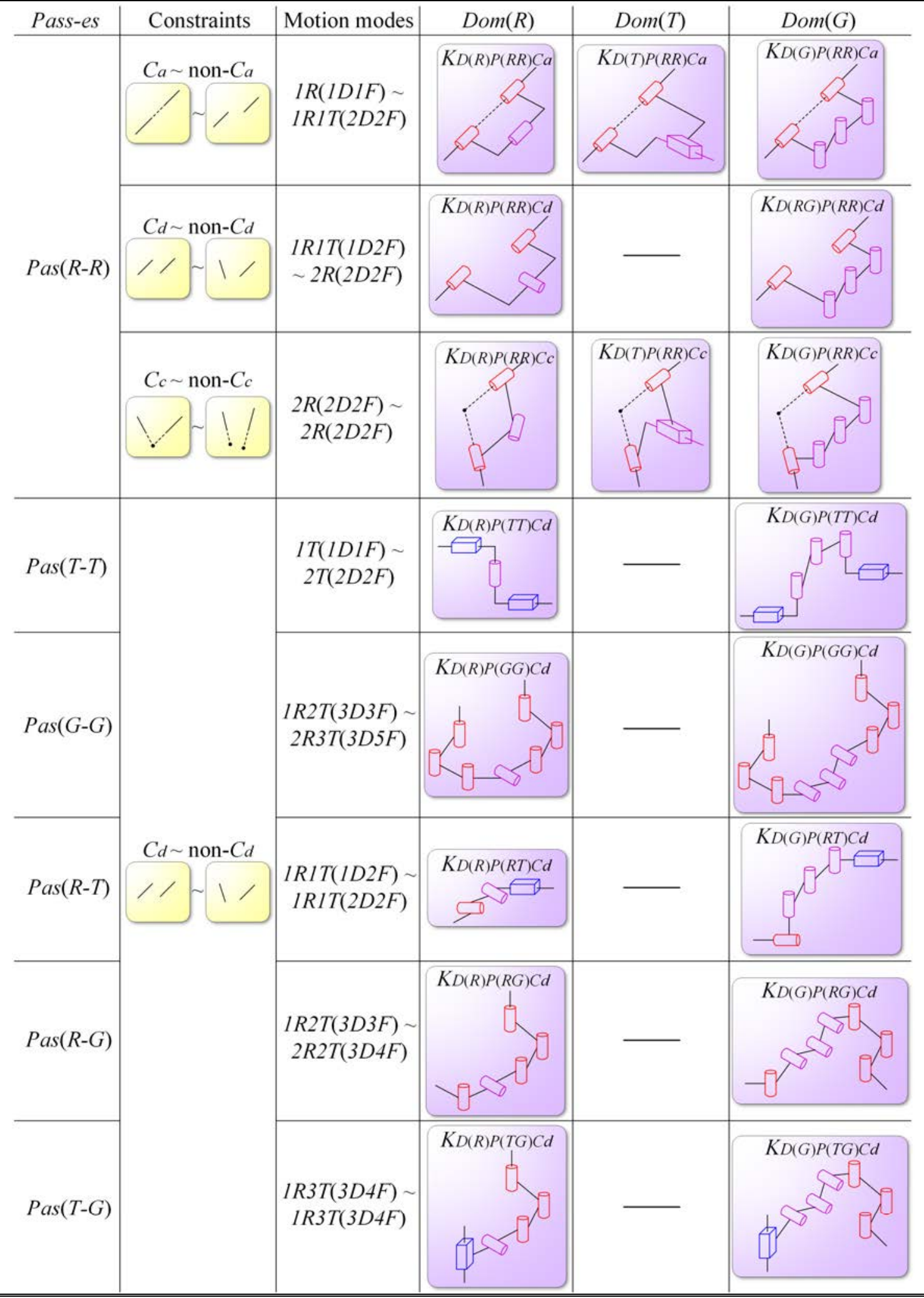

For example, the serial kinematic chain with variable constraint relations $K_{D(T) P(R R) C a}$ can be built by the combination of $\operatorname{Dom}(T)$ and $\operatorname{Pas}(R-R)$ with constraint relations $C_{a}$, where the prismatic joint of $\operatorname{Dom}(T)$ is placed between two revolute joints of $\operatorname{Pas}(R-R)$ and connects the two revolute joints in $C_{a}$ 
constraint relations to form a serial kinematic chain with variable constraint relations. The combinatorial output motion modes of the Pas can be changed from $1 R(1 D 1 F)$ to $1 R 1 T(2 D 2 F)$ when the $C_{a}$ constraint relation of $\operatorname{Pas}(R-R)$ is changed to the non- $C_{a}$ constraint relations by the translational motion of $\operatorname{Dom}(T)$. In Fig. 1, the single loop serial kinematic chain with variable constraint relations is a combination of two serial kinematic chains $K_{D(T) P(R R) C a}$.

In accordance with the method of combinations of Dom-s (3 types) and Pas-es (6 types) with different constraint relations (3 types), there are 18 basic forms of serial kinematic chains with variable constraint relations $K_{*}$ that can be built. All types of motion transformations can be generated by the 18 basic serial kinematic chains. In Table 4, the joints with purple lines are Dom-s, while the joints with red or blue lines are Pas-es.

Based on the basic serial kinematic chains $K_{*}$, the different serial or parallel mechanisms in Table 2. and Table 3. can be combined with the required $K_{*}$ at the chosen detachment points to build the general kinematotropic mechanisms with the related variable motion modes.

\subsection{Design rules for general kinematotropic mechanisms}

In order to design the general kinematotropic mechanisms, the relationships between the displacement subsets of subchains and the variable combinatorial output motions should be determined. Thus, the first design rule about the relations between the input and output motions can be proposed as:

(1) Design rule for relations of input and output motions: The number of 1-DOF joints in a subchain should not be less than the number of DOFs under different independent motion modes, namely, the output displacement subsets of different independent motion modes are the subsets of each subchain.

Since the relationships between variable output motion modes and motions of Dom-s and Pas-es are critical to the design of kinematotropic mechanisms, a second design rule should be observed as:

(2) Design rule for relations of output motion modes and functional objects: The different variable combinatorial output motions of Dom-s and Pas-es are corresponding to the different output motion modes, namely the different output motion modes of the designed kinematotropic mechanisms are corresponding to the different output motion modes of the adopted basic serial kinematic chains with variable constraint relations.

A systematic design method of general kinematotropic mechanisms can be developed based on the design rules, different serial and parallel mechanisms, basic serial kinematic chains with variable constraint relations and the related structural properties.

\section{Design method of general kinematotropic mechanisms}

In order to design the structures of general kinematotropic mechanisms, the related design method should be developed based on the above definitions and analyses. Thus, the systematic design steps and the logical relations can be given as follows: 
(1) Determination of the type of motion transformation $x_{1} R x_{2} T\left(x_{3} D\left(x_{1}+x_{2}\right) F\right) \leftrightarrow$ $x_{4} R x_{5} T\left(x_{6} D\left(x_{4}+x_{5}\right) F\right)$, including the required reference frame. The different variable output motion modes are obtained based on the practical design requirements from Table 1. Any two or more motion modes can be designed in a kinematotropic mechanism.

(2) Corresponding to the types of motion transformations, the independent structures of the different serial chains are determined under the different motion modes. The related serial chains are chosen from Table 2.

(3) Determination of the basic structural form of the kinematotropic mechanism, i.e., the number of subchains and moving platforms can be determined according to the requirements of the practical structural design, and related applied properties.

(4) The parallel mechanisms in Table 3. can be selected in accordance with the types of motion transformation. The independent structures of the different parallel chains are determined by the basic serial/parallel chains, where the parallel chains have the same structure which is derived from the related serial chains.

(5) Determination of the constraint forms of the kinematotropic mechanism, namely of the selftransforming or inter-transforming form.

(6) Determination of the basic constraint relations applied in the motion transformation according to the types of motion transformations and constraint conditions in the chosen parallel chains.

(7) Determination of the types of Dom-s and Pas-es, namely, of the structures of the functional objects according to the structures of the chosen parallel chains.

(8) Determination of different combinatorial forms and constraint relations of Dom-s and Pas-es under different motion modes, i.e., the determination of serial kinematic chains with variable constraint relations, where the basic serial kinematic chains can be chosen from Table 4.

(9) Determination of the detachment points of subchains for the different parallel chains. The detachment points are chosen between the adjacent joints of the chosen constraint relations. In a self-transforming form, the detachment points are determined in subchains, and the variable constraints are defined in the subchains. In an inter-transforming form, the detachment points are determined around the moving platforms, and the variable constraints are defined between the subchains.

(10) Determination of the intersection sets of motions [31, 32] between different motion modes, which can be considered as displacement subsets of all subchains.

(11) Determination of the union sets of motions [31, 32] between different motion modes. Then, the complement of the union sets can be obtained in the displacement sets of spatial motions, which can be considered as common constraints of motions in all subchains.

(12) In accordance with the above choices of functional objects and detachment points of the subchains, the determined parallel chains in different motion modes can be detached respectively and combined each other. Meanwhile the chosen serial kinematic chains with variable constraint relations can be added at the detachment points. The redundant joints that have repetitive effects for the type of motion should be removed, and the combination design should comply with the two design rules.

(13) Determination of the state of mobility bifurcation of the structure, namely of its singularity pose of mobility bifurcation when the mechanism transforms from one motion mode to another mode.

(14) In accordance with the combinatorial kinematotropic structures and the state of mobility bifurcation, the constraint conditions of motion modes that are used to change the motion modes are obtained. In other words, the constraint relations of different subchains in the singularity pose of mobility bifurcation can be determined to transform the motion mode of the mechanism. 
(15) Optimization of the layout of joints and subchains to realize the required design of kinematotropic mechanism.

Based on the displacement group theory, the related operations and design can be determined by the intersection and union sets of displacement group between different motion modes of subchains. The continuous motions of a motion mode are existing under the determined constraint conditions with group operations. The motion mode can be changed by the variable constraint conditions when the structure passes through the singularity pose with different constraint conditions and different results of group operations. The finite motions of the synthesized mechanisms in a determined motion mode can be guaranteed by the corresponding constraint relations.

In light of the above design steps, the logical relations of the systematic method for structural synthesis of general kinematotropic mechanisms is illustrated in Fig. 4., where the cyan parts represent the determination of the basic initial elements, the light blue parts represent the structural design of the basic elements, the light green part is the design rules, and the orange part represents the results of the structural design of kinematotropic mechanisms.

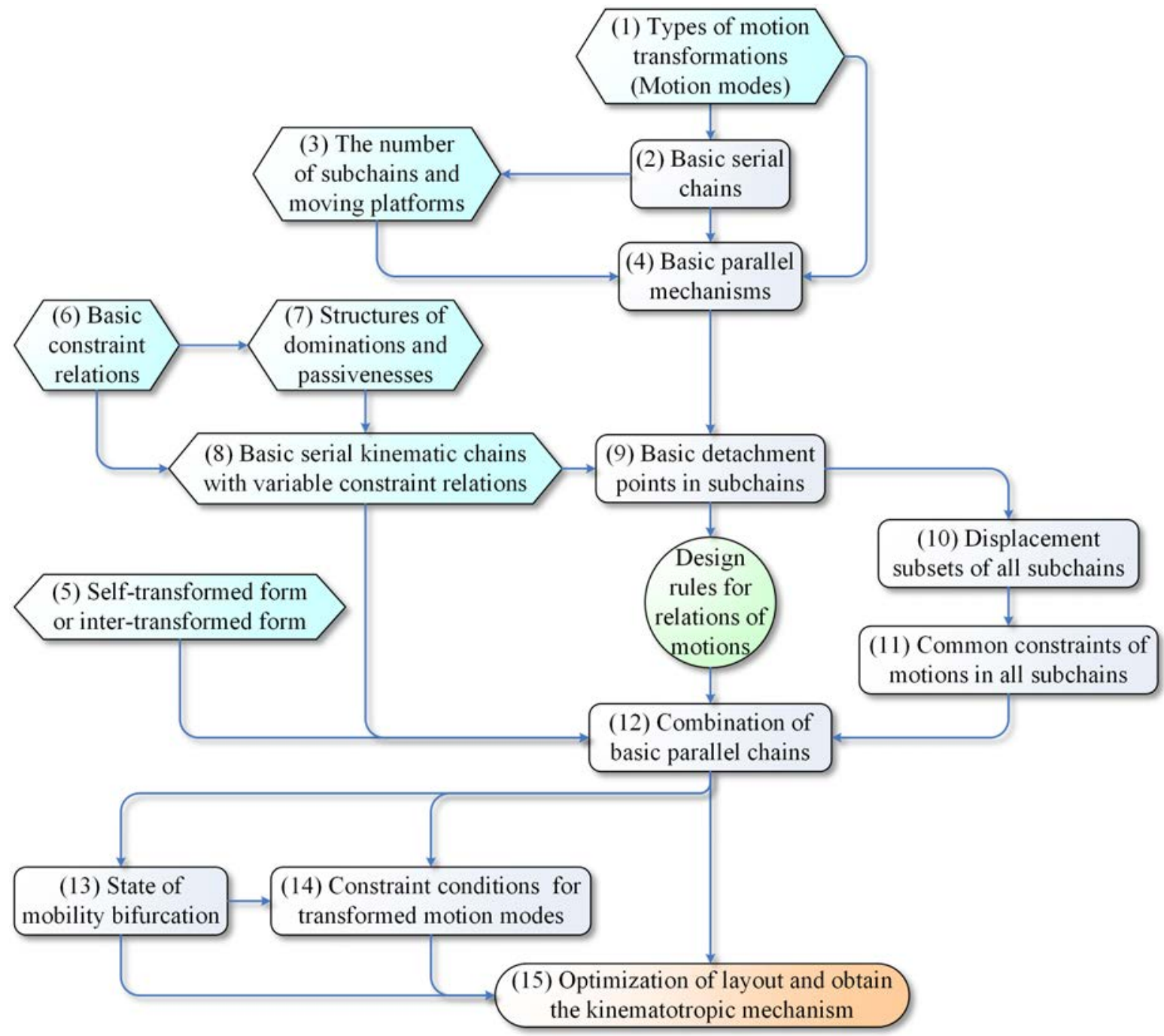

Fig. 4. Logical relations between the design steps of kinematotropic mechanisms 


\section{Examples for the design method of general kinematotropic mechanisms}

In order to demonstrate the applicability of the presented design method, two types of motion transformations with four kinematotropic mechanisms are developed as the typical examples. The related structural design and analysis are illustrated in detail below.

\subsection{Kinematotropic mechanisms with motion transformation $3 R 1 T_{z}(3 D 4 F) \leftrightarrow 1 R_{z} 3 T(3 D 4 F)$}

\subsubsection{Design process of the kinematotropic mechanisms}

In accordance with the design method of general kinematotropic mechanism, the first step of the design is determination of the type of motion transformation in light of the specific requirements. In this example, two motion modes $3 R 1 T_{z}(3 D 4 F)$ and $1 R_{z} 3 T(3 D 4 F)$ are arbitrarily chosen as the type of motion transformation, where the subscript of $T$ is $z$ axis in a fixed reference frame $O-x y z$, which represent that the moving direction of the translation is along the $z$ axis. The same subscript express is applied for the follows.

Corresponding to the two motion modes, the serial chains $S C_{3 R 1 T(3)}$ and $S C_{1 R 3 T(3)}$ can be chosen from Table 2., and the related parallel chains $P C_{3 R I T(2)}$ and $P C_{1 R 3 T(2)}$ are chosen from Table 3. as the basic structures. The structure form is determined as a parallel form with a single moving platform and four subchains. Based on the chosen parallel chains, the basic constraint relations can be determined as $C_{a}$ and $C_{c}$, and the types of Dom-s and Pas-es are determined as $\operatorname{Dom}(G)$ and $P a(R-R)$. Correspondingly, the basic serial kinematic chains with variable constraint relations $K_{D(G) P(R R) C a}$ and $K_{D(G) P(R R) C C}$ are be chosen from Table 4., and the variable motion modes are $1 R(1 D 1 F) \leftrightarrow 1 R 1 T(2 D 2 F)$ and $2 R(2 D 2 F) \leftrightarrow 2 R(2 D 2 F)$, respectively.

In order to achieve the kinematotropic mechanisms with two motion modes $3 R 1 T_{z}(3 D 4 F)$ and $1 R_{z} 3 T(3 D 4 F)$, the two chosen parallel chains should be detached respectively and combined each other. Meanwhile, the chosen serial kinematic chains with variable constraint relations can be added at the detachment points. The detachment points are chosen between the adjacent joints of the chosen constraint relations. The intersection sets of motions between the two motion modes are $T_{z}$ and $R_{z}$. The combination design comply with the two design rules, and the redundant joints should be removed.

In light of the influence range of Dom-s and the above results, two kinematotropic mechanisms corresponding to the two types of constraint forms (self-transforming and inter-transforming forms) can be obtained, respectively. These are shown in Fig. 5., where the red and green dots are the detachment points for self-transforming form and inter-transforming form, respectively. The joints with purple lines are Dom-s which can be actuated to change the state of structure between the two motion modes. 


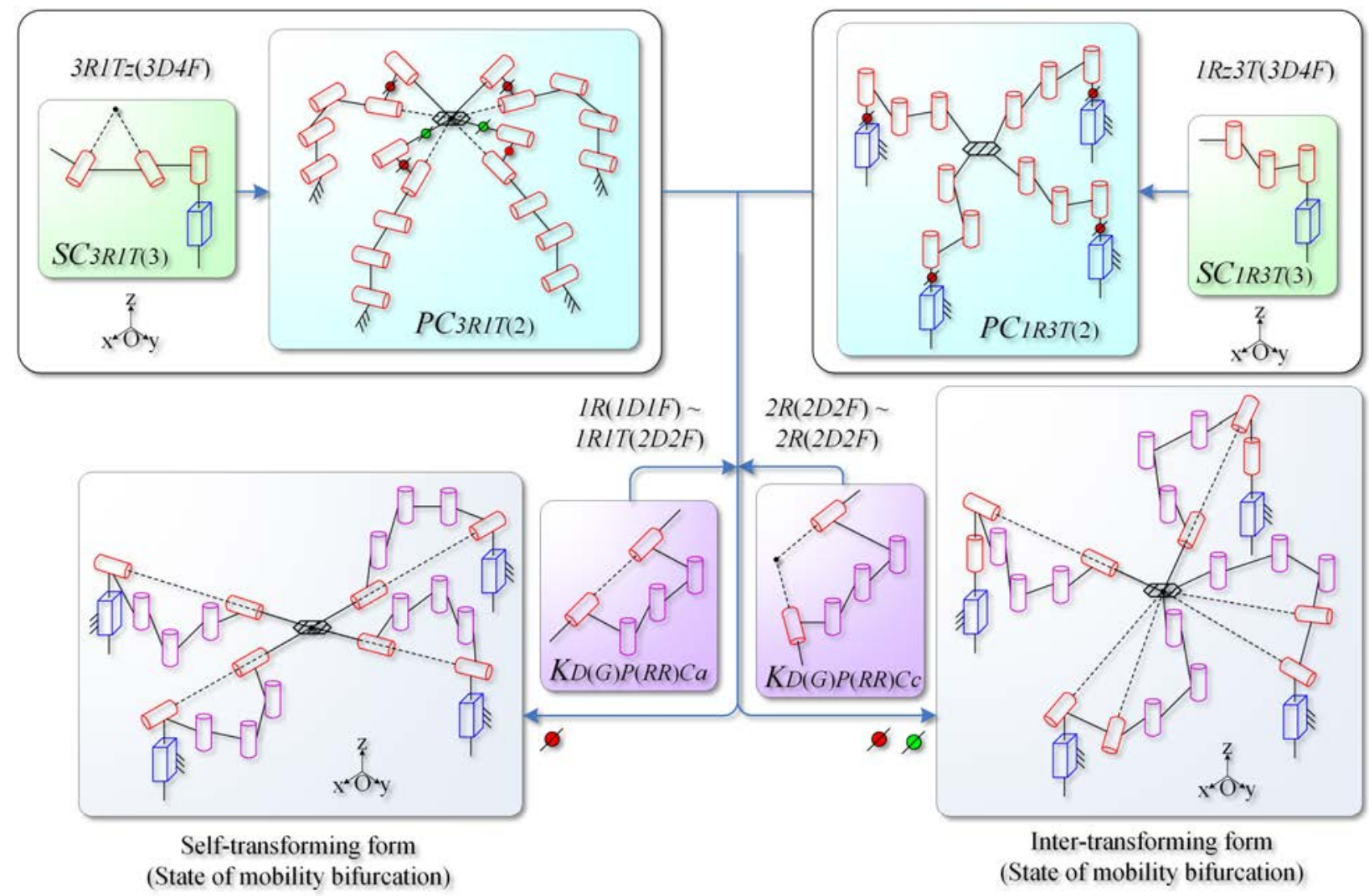

Fig. 5. Two kinematotropic mechanisms with motion transformation $3 R 1 T_{z}(3 D 4 F) \leftrightarrow 1 R_{z} 3 T(3 D 4 F)$

\subsubsection{Variable mobility analysis of the kinematotropic mechanism in the self-transforming form}

The state of the mobility bifurcation of the self-transforming form and the related two motion modes are shown in Fig. 6., where $S_{i}$ and $P_{O}$ represent subchain $i$ and the output moving platform, respectively. Symbols $r_{i j}$ and $t_{i j}$ represent the $j^{\text {th }}$ revolute and prismatic joint in the $i^{\text {th }}$ subchain $S_{i}$, respectively. $O^{\prime}$ is the intersection point of axes $r_{i 1}$, and $O^{\prime \prime}$ is the intersection point of axes $r_{i 5} . \operatorname{Dom}(G)_{i}$ contains the displacement subset $\{G\}$ that consists of $r_{i 2}, r_{i 3}$ and $r_{i 4}$. Pas $(R-R)_{i}$ consists of $r_{i 1} \leftrightarrow r_{i 5}$, and is distributed in each subchain. The relations of $\operatorname{Pas}(R-R)_{i}$ are affected by $\operatorname{Dom}(G)_{i}$ only in a subchain to transform the motion modes. If: (1) axes $r_{i 1}$ and $r_{i 5}$ are collinear and (2) axes $r_{i 2}, r_{i 3}$ and $r_{i 4}$ are equidirectional with the direction of axis $z$ in the respective subchain, the mechanism is set in the state of mobility bifurcation. 
Self-transforming form

(State of mobility bifurcation)

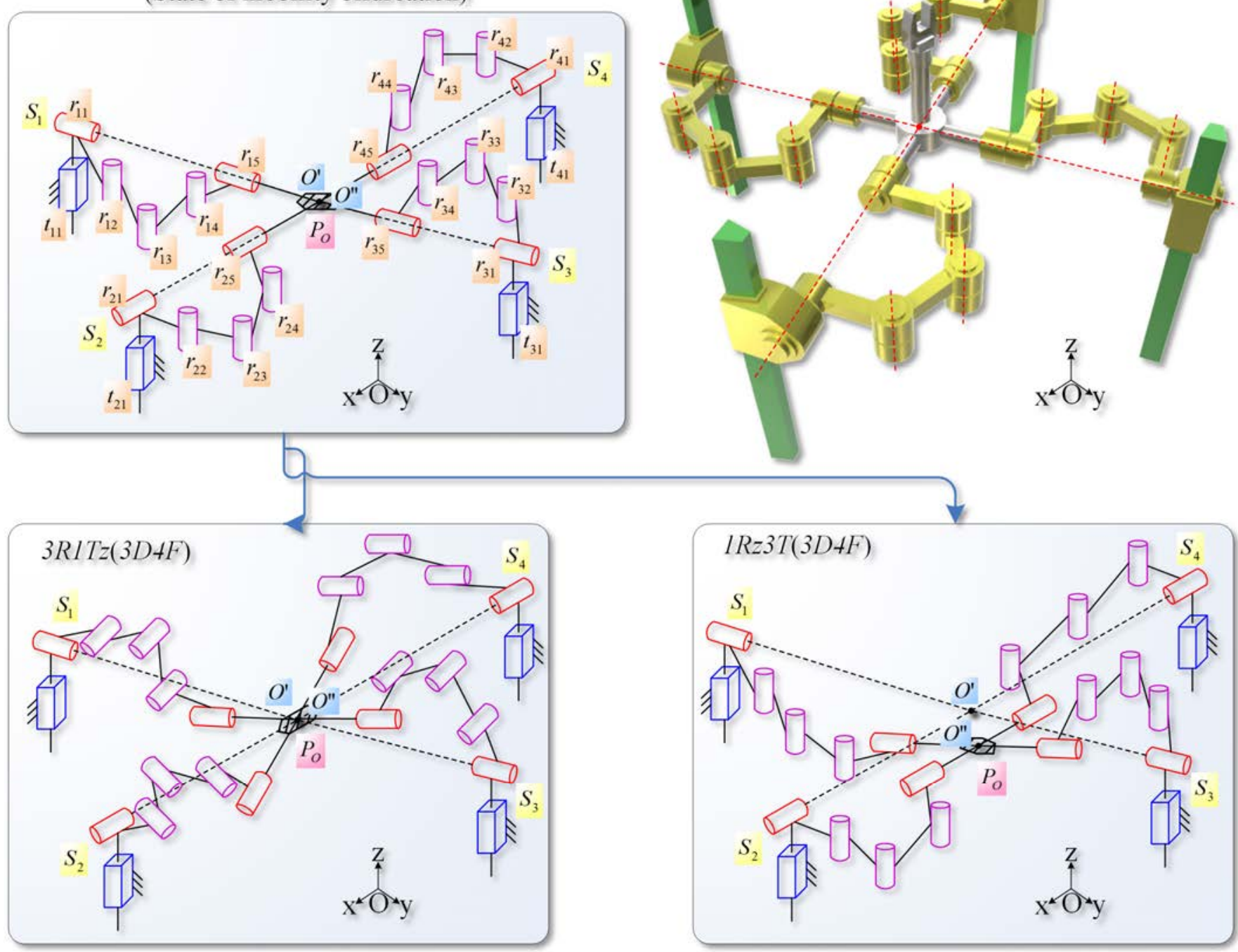

Fig. 6. Motion modes of the kinematotropic mechanism $3 R 1 T_{z}(3 D 4 F) \leftrightarrow 1 R_{z} 3 T(3 D 4 F)$ in the self-transforming form

In the motion mode $3 R 1 T_{z}(3 D 4 F)$, the constraint conditions are: (1) all axes $r_{i 1}$ and $r_{i 5}$ intersect at point $O^{\prime}$, namely points $O^{\prime}$ and $O^{\prime \prime}$ are collinear, (2) axes $r_{i 1}$ and $r_{i 5}$ are non-collinear, and (3) axes $r_{i 2}, r_{i 3}$ and $r_{i 4}$ are non-equidirectional with direction $z$. In the motion mode $1 R_{z} 3 T(3 D 4 F)$, the constraint conditions are: (1) point $O^{\prime \prime}$ is not collinear with point $O^{\prime}$, and (2) axes $r_{i 2}, r_{i 3}$ and $r_{i 4}$ are equidirectional with the direction $z$. Based on the displacement group theory [31, 32], the corresponding intersection operations between the motions of subchains, and the variable output displacement subsets are: 


$$
\begin{aligned}
\left\{P_{O}\right\} & =\bigcap_{i=1}^{4}\left\{T\left(t_{i 1}\right)\right\}\left\{R\left(r_{i 1}\right)\right\}\left\{G\left(r_{i 2}, r_{i 3}, r_{i 4}\right)\right\}\left\{R\left(r_{i 5}\right)\right\} \\
& =\{T(z)\} \bigcap_{i=1,3}\left(\left\{O^{\prime} R(y)\right\}\left\{G\left(r_{i 2}\right)\right\}\left\{O^{\prime \prime} R\left(r_{i 5}\right)\right\}\right) \cap \bigcap_{i=2,4}\left(\left\{O^{\prime} R(x)\right\}\left\{G\left(r_{i 2}\right)\right\}\left\{\left\{^{O \prime \prime} R\left(r_{i 5}\right)\right\}\right)\right. \\
& =\left\{\begin{array}{l}
T\{z\} R\{x, y, z\}, O^{\prime}=O^{\prime \prime}, r_{i 1} \neq r_{i 5}, r_{i 2} \neq z \\
T\{x, y, z\} R\{z\}, O^{\prime} \neq O^{\prime \prime}, r_{i 2}=z
\end{array}\right.
\end{aligned}
$$

where $\left\{P_{O}\right\}$ represent the output displacement subset of moving platform $P_{O}$, while $\{R(*)\},\{T(*)\}$ and $\{G(*)\}$ represent the rotational, translational and planar displacement subsets along the different axes, respectively.

\subsubsection{Variable mobility analysis of the kinematotropic mechanism in the inter-transforming form}

The state of mobility bifurcation of the inter-transforming form and the related two motion modes are shown in Fig. 7., where $O^{\prime}$ is the intersection point of axes $r_{12}, r_{16}, r_{42}$ and $r_{46}$, and $O^{\prime \prime}$ is the intersection point of axes $r_{21}, r_{22}, r_{31}$ and $r_{32}$. $\operatorname{Dom}(G)_{i}$ contains the displacement subset $\{G\}$ that consists of $r_{i 3}, r_{i 4}$ and $r_{i 5}$. Pas $(R-R)_{i}$ consists of $r_{12} \leftrightarrow r_{16}, r_{42} \leftrightarrow r_{46}, r_{22} \leftrightarrow r_{16}$ and $r_{32} \leftrightarrow r_{46}$, which are distributed in the different subchains and around the moving platform. The relations of $\operatorname{Pas}(R-R)_{i}$ are affected by $\operatorname{Dom}(G)_{i}$ between the different subchains to transform the motion modes. If: (1) axes $r_{12}, r_{16}$ and $r_{42}, r_{46}$ are collinear respectively, (2) points $O^{\prime}$ and $O^{\prime \prime}$ are collinear, and (3) axes $r_{i 3}, r_{i 4}$ and $r_{i 5}$ are equidirectional with the direction $z$ in the respective subchain, the mechanism is set in the state of mobility bifurcation.

In the motion mode $3 R 1 T_{z}(3 D 4 F)$, the constraint conditions are: (1) points $O^{\prime}$ and $O^{\prime \prime}$ are collinear, (2) axes $r_{12}, r_{16}$ and $r_{42}, r_{46}$ are non-collinear respectively, and (3) axes $r_{i 3}, r_{i 4}$ and $r_{i 5}$ are nonequidirectional with the direction $z$. In the motion mode $1 R_{z} 3 T(3 D 4 F)$, the constraint conditions are: (1) point $O^{\prime \prime}$ is not collinear with point $O^{\prime}$, and (2) axes $r_{i 2}, r_{i 3}$ and $r_{i 4}$ are equidirectional with the direction $Z$. The corresponding intersection operations between the motions of subchains, and the variable output displacement subsets are:

$$
\begin{aligned}
\left\{P_{O}\right\} & =\bigcap_{i=1,4}\left(\left\{T\left(t_{i 1}\right)\right\}\left\{R\left(r_{i 1}\right)\right\}\left\{R\left(r_{i 2}\right)\right\}\left\{G\left(r_{i 3}, r_{i 4}, r_{i 5}\right)\right\}\left\{R\left(r_{i 6}\right)\right\}\right) \bigcap \bigcap_{i=2,3}\left(\left\{T\left(t_{i 1}\right)\right\}\left\{R\left(r_{i 1}\right)\right\}\left\{R\left(r_{i 2}\right)\right\}\left\{G\left(r_{i 3}, r_{i 4}, r_{i 5}\right)\right\}\right) \\
& =\{T(z)\} \bigcap_{i=1,4}\left(\{R(z)\}\left\{^{O^{\prime}} R\left(r_{i 2}\right)\right\}\left\{G\left(r_{i 3}\right)\right\}\left\{{ }^{\prime} R\left(r_{i 6}\right)\right\}\right) \bigcap \bigcap_{i=2,4}\left(\left\{{ }^{\prime \prime} R\left(r_{i 1}\right)\right\}\left\{^{O^{\prime \prime}} R\left(r_{i 2}\right)\right\}\left\{G\left(r_{i 3}\right)\right\}\right) \\
& =\left\{\begin{array}{l}
T\{z\} R\{x, y, z\}, O^{\prime}=O^{\prime \prime}, r_{12} \neq r_{16}, r_{42} \neq r_{46}, r_{i 3} \neq z \\
T\{x, y, z\} R\{z\}, O^{\prime} \neq O^{\prime \prime}, r_{i 3}=z
\end{array}\right.
\end{aligned}
$$


Inter-transforming form

(State of mobility bifurcation)

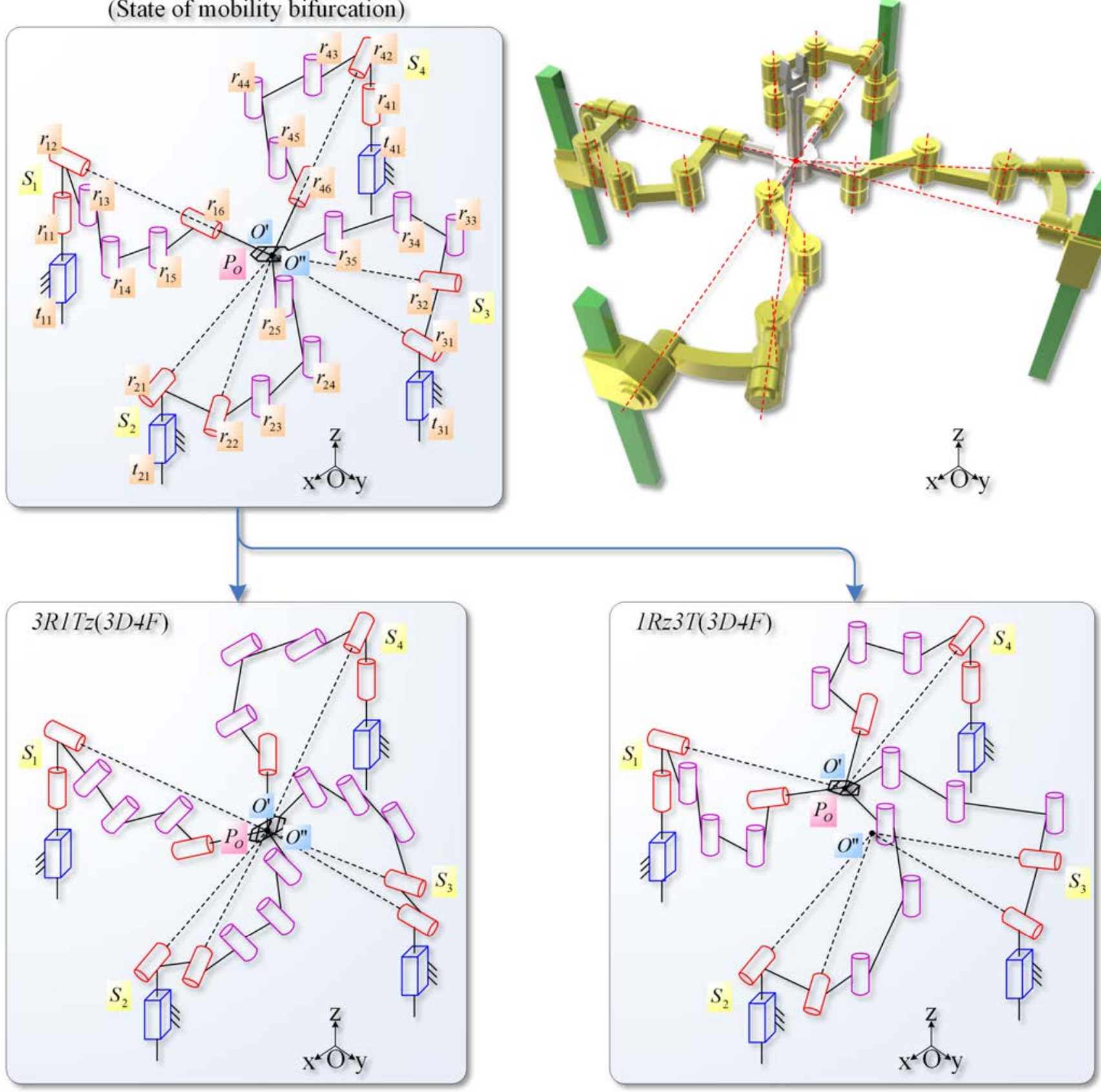

Fig. 7. Motion modes of the kinematotropic mechanism $3 R 1 T_{z}(3 D 4 F) \leftrightarrow 1 R_{z} 3 T(3 D 4 F)$ in the inter-transforming form

\subsection{Kinematotropic mechanisms with motion transformation $1 R_{y} 1 T_{z}(2 D 2 F) \leftrightarrow 1 R_{z} 2 T_{x y}(3 D 3 F)$}

\subsubsection{Design process of the kinematotropic mechanisms}

In this example, two motion modes $1 R_{y} 1 T_{z}(2 D 2 F)$ and $1 R_{z} 2 T_{x y}(3 D 3 F)$ are chosen as the type of motion transformation. Correspondingly, the related serial chains $S B_{1 R 1 T(2)}$ and $S B_{1 R 2 T}$ can be chosen from Table 2., and the related parallel chains $P C_{1 R 1 T(2 D)(2)}$ and $P B_{1 R 2 T}$ are chosen from Table 3. as the basic structures. The structure form is determined as a parallel form with a single moving platform and three subchains. 
Based on the chosen parallel chains, the basic constraint relations are determined as $C_{a}$ and $C_{d}$, and the types of Dom-s and Pas-es can be determined as $\operatorname{Dom}(R)$ and $\operatorname{Pa}(R-R)$. Meanwhile, the basic serial kinematic chains with variable constraint relations $K_{D(R) P(R R) C a}$ and $K_{D(R) P(R R) C d}$ are chosen from Table 4., and the variable motion modes are $1 R 1 T(2 D 2 F) \leftrightarrow 2 R(2 D 2 F)$ and $1 R(1 D 1 F) \leftrightarrow 1 R 1 T(2 D 2 F)$, respectively.

In accordance with design method, the chosen parallel chains and serial kinematic chains with variable constraint relations can be combined at the detachment points. There are no intersection sets of motions between the two motion modes. The combination design comply with the two design rules, and the redundant joints are removed. Based on the influence range of Dom-s and the related results, two kinematotropic mechanisms corresponding to the two types of constraint forms (self-transforming and inter-transforming forms) can be obtained, respectively. The obtained structures are shown in Fig. 8., where the joints with purple lines are Dom-s which are actuated to change the state of structure between the two motion modes.

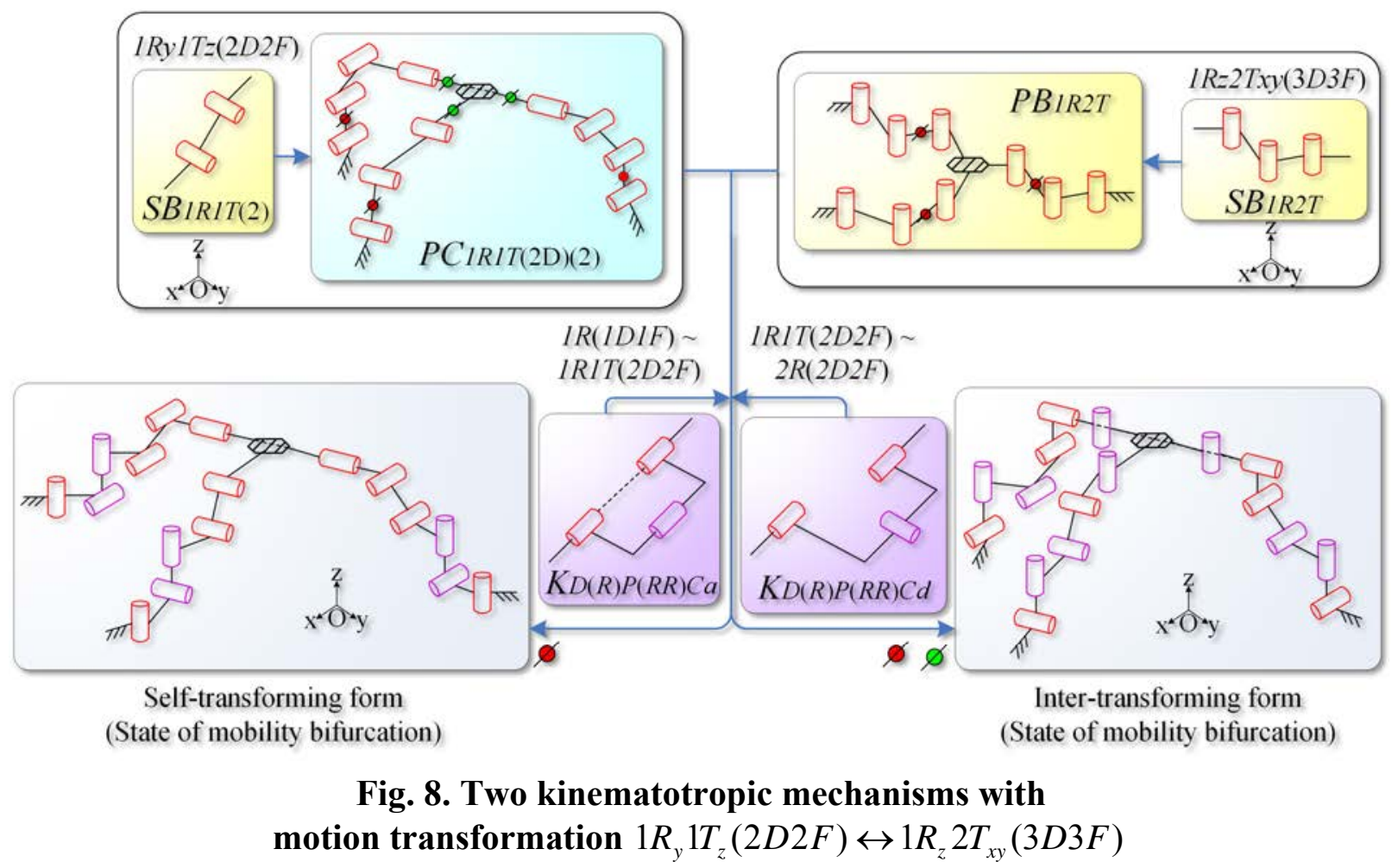

\subsubsection{Variable mobility analysis of the kinematotropic mechanism in the self-transforming form}

The state of mobility bifurcation of the self-transforming form and the related two motion modes are shown in Fig. 9., where $\operatorname{Dom}(R)_{i}$ consists of two orthogonal $r_{i 2}$ and $r_{i 3}$. Pas $(R-R)_{i}$ contains $r_{i 1} \leftrightarrow r_{i 3}$ and $r_{i 2} \leftrightarrow r_{i 4}$, and is distributed in each subchain. If: (1) axes $r_{i 1}$ and $r_{i 3}$ are equidirectional and (2) axes $r_{i 2}$ and $r_{i 4}$ are equidirectional in the respective subchain, the mechanism is set in the state of mobility bifurcation.

In the motion mode $1 R_{y} 1 T_{z}(2 D 2 F)$, the constraint conditions are: (1) the axes $r_{i 1}$ and $r_{i 3}$ are nonequidirectional, and (2) axes $r_{i 2}$ and $r_{i 4}$ are equidirectional, where $r_{i 2}$ are Dom-s. In the motion mode 
$1 R_{z} 2 T_{x y}(3 D 3 F)$, the constraint conditions are: (1) the axes $r_{i 1}$ and $r_{i 3}$ are equidirectional, and (2) axes $r_{i 2}$ and $r_{i 4}$ are non-equidirectional, where $r_{i 3}$ are Dom-s. The corresponding intersection operations between the motions of subchains, and the variable output displacement subsets are:

Self-transforming form (State of mobility bifurcation)

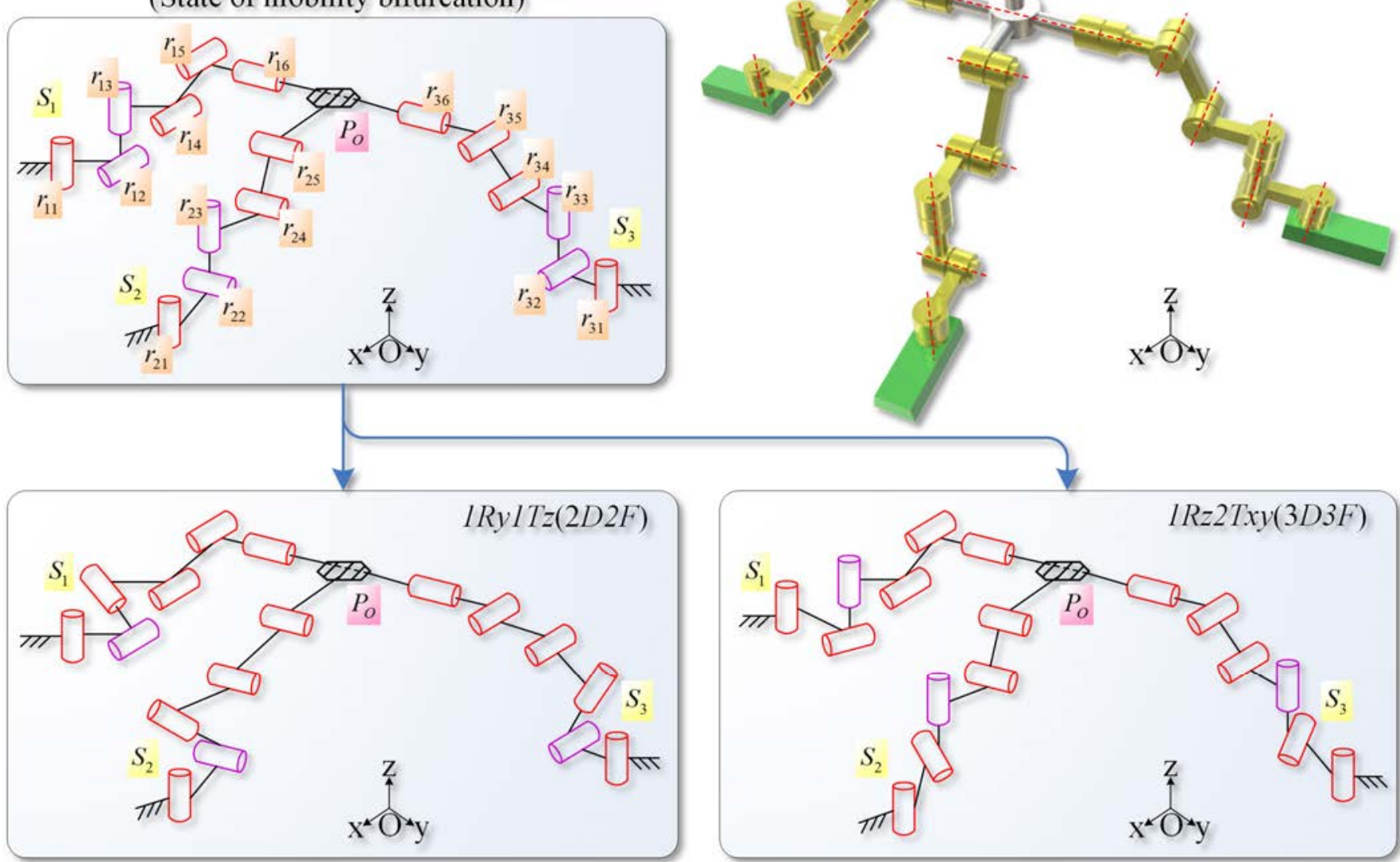

Fig. 9. Motion modes of the kinematotropic mechanism $1 R_{y} 1 T_{z}(2 D 2 F) \leftrightarrow 1 R_{z} 2 T_{x y}(3 D 3 F)$ in the self-transforming form

$$
\begin{aligned}
\left\{P_{O}\right\}= & \bigcap_{i=1,3}\left(\left\{R\left(r_{i 1}\right)\right\}\left\{R\left(r_{i 2}\right)\right\}\left\{R\left(r_{i 3}\right)\right\}\left\{R\left(r_{i 4}\right)\right\}\left\{R\left(r_{i 5}\right)\right\}\left\{R\left(r_{i 6}\right)\right\}\right) \cap \\
& \left(\left\{R\left(r_{21}\right)\right\}\left\{R\left(r_{22}\right)\right\}\left\{R\left(r_{23}\right)\right\}\left\{R\left(r_{24}\right)\right\}\left\{R\left(r_{25}\right)\right\}\right) \\
= & \bigcap_{i=1,3}\left(\{R(z)\}\left\{R\left(r_{i 2} \perp z\right)\right\}\left\{R\left(r_{i 3} \perp r_{i 2}\right)\right\}\left\{R\left(r_{i 4} \perp r_{i 3}\right)\right\}\left\{R\left(r_{i 5} / / r_{i 4}\right)\right\}\left\{R\left(r_{i 6} \perp r_{i 5}\right)\right\}\right) \cap \\
& \left(\{R(z)\}\left\{R\left(r_{22} \perp z\right)\right\}\left\{R\left(r_{23} \perp r_{22}\right)\right\}\left\{R\left(r_{24} \perp r_{23}\right)\right\}\left\{R\left(r_{25} / / r_{24} / / r_{16}=r_{36}\right)\right\}\right) \\
= & \left\{\begin{array}{l}
R\{y\} T\{z\}, r_{i 1} \angle r_{i 3}, r_{i 2} / / r_{i 4} \\
R\{z\} T\{x, y\}, r_{i 1} / / r_{i 3}, r_{i 2} \angle r_{i 4}
\end{array}\right.
\end{aligned}
$$

\subsubsection{Variable mobility analysis of the kinematotropic mechanism in the inter-transforming form}

The state of mobility bifurcation of the inter-transforming form and the related two motion modes are shown in Fig. 10., where $\operatorname{Dom}(R)_{i}$ consists of $r_{i 2}, r_{i 3}, r_{16}, r_{25}$ and $r_{36}$. $\operatorname{Pas}(R-R)_{i}$ that consists of 
$r_{12} \leftrightarrow r_{16}, r_{22} \leftrightarrow r_{25}, r_{32} \leftrightarrow r_{36}, r_{i 1} \leftrightarrow r_{i 3}, r_{15} \leftrightarrow r_{35}, r_{15} \leftrightarrow r_{24}$ and $r_{24} \leftrightarrow r_{35}$, which are distributed in the different subchains and around the moving platform. If: (1) axes $r_{12}, r_{16}, r_{22}, r_{25}, r_{32}$ and $r_{36}$ are equidirectional with direction $z$, (2) axes $r_{11}, r_{13}, r_{31}, r_{33}$ are equidirectional with direction $x$, (3) axes $r_{21}, r_{23}$ are equidirectional with direction $y$, and (4) axes $r_{15}, r_{35}$ are collinear, the mechanism is set in the state of mobility bifurcation.

In the motion mode $1 R_{y} 1 T_{z}(2 D 2 F)$, the constraint conditions are: (1) axes $r_{12}, r_{16}, r_{22}, r_{25}, r_{32}$ and $r_{36}$ are non-equidirectional, (2) axes $r_{11}, r_{13}, r_{31}, r_{33}$ are equidirectional with direction $x$, (3) axes $r_{21}, r_{23}$ are equidirectional with direction $y$, and (4) axes $r_{15}, r_{35}$ are collinear. The joints with axis $r_{12}, r_{16}, r_{22}, r_{25}$, $r_{32}$ and $r_{36}$ are locked, while the remaining joints are effective joints. In the motion mode $1 R_{z} 2 T_{x y}(3 D 3 F)$, the constraint conditions are: (1) axes $r_{12}, r_{16}, r_{22}, r_{25}, r_{32}$ and $r_{36}$ are equidirectional with direction $z,(2)$ axes of $r_{11}, r_{13}, r_{31}, r_{33}$ are non-equidirectional, (3) axes $r_{21}, r_{23}$ are non-equidirectional, and (4) axes $r_{15}$, $r_{35}$ are non-collinear. The joints with axis $r_{11}, r_{15}, r_{21}, r_{31}$ and $r_{35}$ are locked, while the remaining joints are effective joints. The corresponding intersection operations between the motions of subchains, and the variable output displacement subsets are:

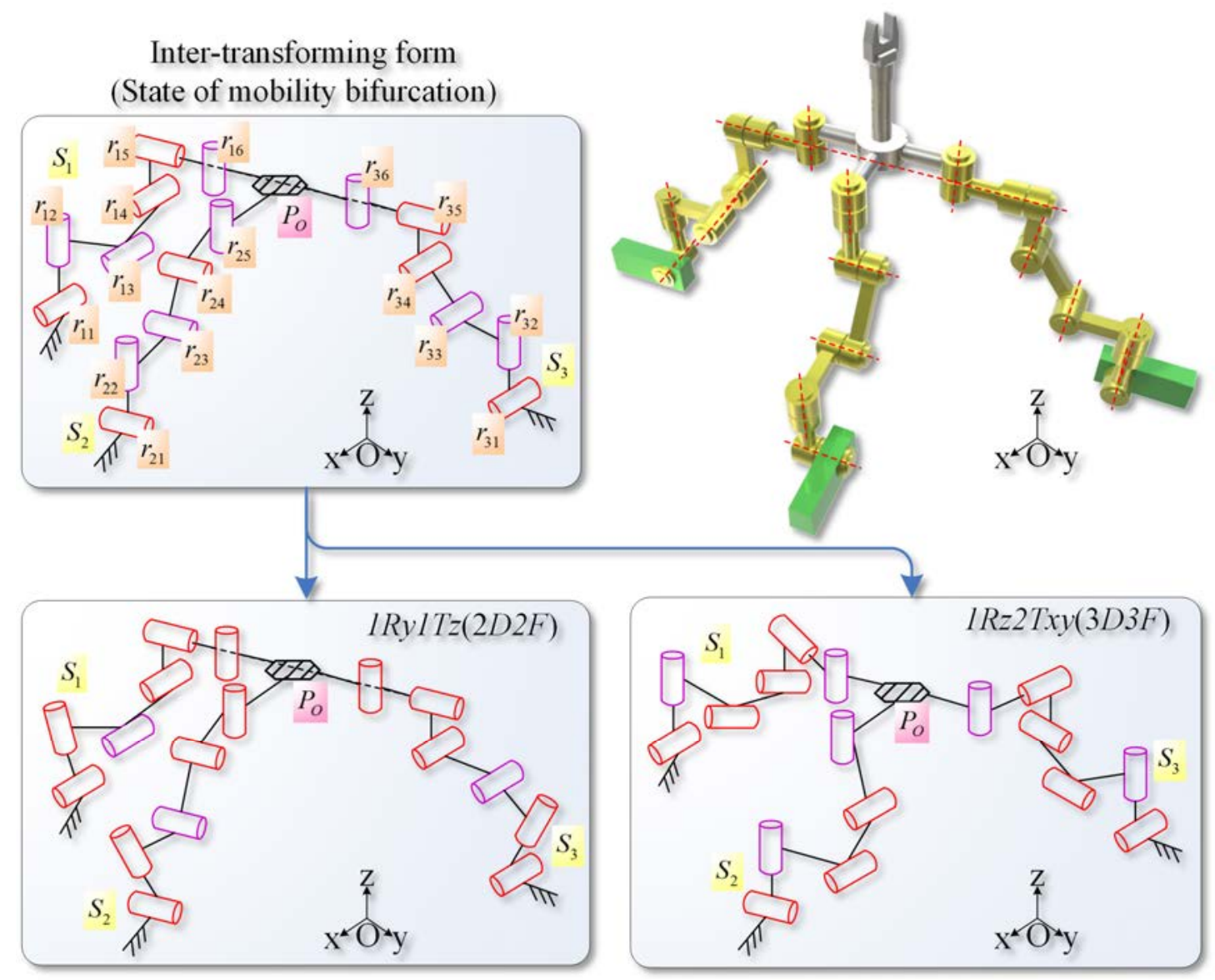

Fig. 10. Motion modes of the kinematotropic mechanism $1 R_{y} 1 T_{z}(2 D 2 F) \leftrightarrow 1 R_{z} 2 T_{x y}(3 D 3 F)$ in the inter-transforming form 


$$
\begin{aligned}
\left\{P_{O}\right\}= & \bigcap_{i=1,3}\left(\left\{R\left(r_{i 1}\right)\right\}\left\{R\left(r_{i 2}\right)\right\}\left\{R\left(r_{i 3}\right)\right\}\left\{R\left(r_{i 4}\right)\right\}\left\{R\left(r_{i 5}\right)\right\}\left\{R\left(r_{i 6}\right)\right\}\right) \cap \\
& \left(\left\{R\left(r_{21}\right)\right\}\left\{R\left(r_{22}\right)\right\}\left\{R\left(r_{23}\right)\right\}\left\{R\left(r_{24}\right)\right\}\left\{R\left(r_{25}\right)\right\}\right) \\
= & \bigcap_{i=1,3}\left(\{R(x)\}\left\{R\left(r_{i 2} \perp x\right)\right\}\left\{R\left(r_{i 3} \perp r_{i 2}\right)\right\}\left\{R\left(r_{i 4} / / r_{i 3}\right)\right\}\left\{R\left(r_{i 5} \perp r_{i 4}\right)\right\}\left\{R\left(r_{i 6} \perp r_{i 5}\right)\right\}\right) \cap \\
& \left(\{R(y)\}\left\{R\left(r_{22} \perp y\right)\right\}\left\{R\left(r_{23} \perp r_{22}\right)\right\}\left\{R\left(r_{24} / / r_{23}\right)\right\}\left\{R\left(r_{25} / / r_{16} / / r_{36} \perp r_{24}\right)\right\}\right) \\
= & \left\{\begin{array}{l}
R\{y\} T\{z\}, r_{12} \angle r_{22} \angle r_{32} \angle r_{25}, r_{13} / / r_{33} / / x, r_{23} / / y, r_{15}=r_{35} \\
R\{z\} T\{x, y\}, r_{12} / / r_{22} / / r_{32} / / r_{25} / / z, r_{13} \angle r_{33} \angle x, r_{23} \angle y, r_{15} \angle r_{35}
\end{array}\right.
\end{aligned}
$$

\section{Conclusions}

This paper presents a method for the structural synthesis of general kinematotropic mechanisms. By considering the combination design method, the basic mechanisms in serial and parallel forms are adopted. The basic kinematotropic properties and the related design rules of the general kinematotropic mechanisms are systematically proposed, which include the characteristics and essence of the kinematotropic property, constraint relations and the basic serial kinematic chains with variable constraint relations. A design method and the related logical relations are presented in 15 steps. At last, two types of motion transformations with four kinematotropic mechanisms are synthesized to verify the applicability of the design method. Meanwhile, the related variable mobility of the four kinematotropic mechanisms are analyzed in details.

In the process of design, the results are not unique in light of the initial choice of related serial or parallel chains, types of dominations and passivenesses, constraint relations and the basic serial kinematic chains with variable constraint relations. Therefore, an optimization of the initial conditions can be developed in accordance with the practical requirements. Moreover, in future research, three or more motion modes of the type of motion transformation can be developed, and the related different spatial motion elements such as spherical, helical, conical, etc. motions should be included with spherical/helical joints, also by considering the specific geometry of linkages/joints with variable topologies, to design the more general kinematotropic mechanism with the reinforced variable motion abilities.

\section{Acknowledgments}

The authors gratefully acknowledge the supports of the U.S. National Science Foundation under grant DMI-0600175, and the U.S. Department of Energy DE-EE0005764.

\section{Nomenclature}

$O-x y z-$ reference frame of the fixed platform

$R$ - rotational motion

$T$ - translational motion

$G$ - planar motion

$D$ - motion axis

$F$ - degree of freedom

$S B_{*}$ - basic serial chain

$S C_{*}$ - serial chain combined by basic serial chains 
$P B_{*}$ — basic parallel chain

$P C_{*}$ - parallel chain combined by basic parallel chains

Dom - domination

Pas - passiveness

$\operatorname{Dom}\left(n_{i}\right)$ - axis of domination $n_{i}$

$\operatorname{Pas}\left(n_{i}\right)$ - axis of passiveness $n_{i}$

$\operatorname{Dom}(R)$ - type of domination

$\operatorname{Pas}(R-R)$ - type of passiveness

$C_{a}$ - axes of joints are collinear

$C_{d}$ - axes or directions of joints are equidirectional

$C_{c}$ - axes of joints are intersecting at a point

$K_{*}$ - basic serial kinematic chains with variable constraint relations

$S_{i}$ - subchain $i$

$P_{O}$ - output moving platform

$r_{i j}$ - the $j^{\text {th }}$ revolute joint in the $i^{\text {th }}$ subchain $S_{i}$

$t_{i j}$ - the $j^{\text {th }}$ prismatic joint in the $i^{\text {th }}$ subchain $S_{i}$

$O^{\prime}, O^{\prime \prime}$ - intersection points of joints axes

$\left\{P_{O}\right\}$ - output displacement subset of moving platform

$\{R(*)\}$ - rotational displacement subset

$\{T(*)\}$ - translational displacement subset

$\{G(*)\}$ - planar displacement subset

\section{References}

[1] Wohlhart, K., 1996, "Kinematotropic mechanisms," Kluwer Academic Publishers, Dordrecht, Recent Advances in Robot Kinematics, pp. 359-368.

[2] Roeschel, O., 2000, "Moebius mechanisms," Kluwer Academic Publishers, Dordrecht, Recent Advances in Robot Kinematics, pp. 375-382.

[3] Galletti, C., and Giannotti, E., 2002, "Multiloop kinematotropic mechanisms," Proceedings of ASME Design Engineering Technical Conference, Montreal, Canada, DETC2002/MECH-34251.

[4] Zlatanov, D., Bonev, I. A., and Gosselin, C. M., 2002, "Constraint Singularities as C-Space Singularities," Advances in Robot Kinematics Theory and Applications, Kluwer Academic Publishers, Dordrecht, pp. 183192.

[5] Galletti, C., and Fanghella, P., 2001, "Single-loop kinematotropic mechanisms," Mech. Mach. Theory, 36, pp. 743-761.

[6] Galletti, C., and Fanghella, P., 1999, "Kinematotropic properties and pair connectivities in single-loop spatial mechanisms," in Proc. 10th World Congr. Theory Mach. Mechanisms, Oulu, Finland, pp. 560-565.

[7] Fanghella, P., Galletti, C., and Giannotti, E., 2006, "Parallel robots that change their group of motion," Advances in Robot Kinematics, The Netherlands: Springer, pp. 49-56.

[8] Refaat, S., Hervé, J. M., Nahavandi, S., and Trinh, H., 2007, "Two-mode over-constrained three-dofs rotational-translational linear-motor-based parallel-kinematics mechanism for machine tool applications," Robotica, 25, pp. 461-466.

[9] Kong, X. W., Gosselin, C. M., and Richard, P. L., 2007, "Type Synthesis of Parallel Mechanisms With Multiple Operation Modes," ASME J. Mech. Des., 129, pp. 595-601.

[10] Li, Q. C., and Hervé, J. M., 2009, "Parallel Mechanisms with Bifurcation of Schoenflies Motion," IEEE 
Transactions on Robotics, 25, pp. 158-164.

[11] Gogu, G., 2011, "Maximally Regular T2R1-Type Parallel Manipulators with Bifurcated Spatial Motion," ASME J. Mech. Rob., 3, pp. 011010.

[12] Lee, C. C., and Hervé, J. M., 2004, "Synthesis of two kinds of discontinuously movable spatial 7R mechanisms through the group algebraic structure of displacement set," Proceedings of 11th IFToMM Word Congress, Tianjin, China, pp. 197-201.

[13] Lee, C. C., and Hervé, J. M., 2005, "Discontinuously movable seven-link mechanisms via group-algebraic approach," Journal of Mechanical Engineering Science, Proceedings of the Institution of Mechanical Engineers, 219, pp. 577-587.

[14] Lee, C. C., and Hervé, J. M., 2007, "Discontinuously movable 8R mechanisms with an infinity of bifurcations," Proceedings of 12th IFToMM Word Congress, Besancon, France.

[15] Balli, S. S., and Chand, S., 2003, "Synthesis of a planar seven-link mechanism with variable topology for motion between two dead-center positions," Mech. Mach. Theory, 38, pp. 1271-1287.

[16] Balli, S. S., and Chand, S., 2004, "Synthesis of a five-bar mechanism of variable topology type with transmission angle control," ASME J. Mech. Des., 126, pp. 128-134.

[17] Yan, H. S., and Kang, C. H., 2009, "Configuration synthesis of mechanisms with variable topologies," Mech. Mach. Theory, 44, pp. 896-911.

[18] Kuo, C. H., and Yan, H. S., 2007, "On the Mobility and Configuration Singularity in Mechanisms with Variable Topologies," ASME J. Mech. Des., 129, pp. 617-624.

[19] Gan, D., Dai, J. S., and Caldwell, D., 2011, "Constraint-Based Limb Synthesis and Mobility-Change-Aimed Mechanism Construction,” ASME J. Mech. Des., 133, pp. 051001.

[20] Kong, X. W., 2011, "Type Synthesis of 3-DOF Parallel Manipulators with Both Planar and Spatial Translational Operation Modes," ASME 2011 International Design Engineering Technical Conferences, 28-31 August 2011, Washington, DC, USA, Paper No. DETC2011-48510.

[21] Yan, H. S., and Kuo, C. H., 2006, "Topological Representations and Characteristics of Variable Kinematic Joints,” ASME J. Mech. Des., 128, pp. 384-391.

[22] Dai, J. S., and Jones, J. R., 1998, "Mobility in metamorphic mechanisms of foldable/erectable kinds," Proceedings of ASME Design Engineering Technical Conferences, Atlanta, Georgia, DETC98/MECH-5902.

[23] Dai, J. S., and Jones, J. R., 1999, "Mobility in metamorphic mechanisms of foldable/erectable kinds," ASME J. Mech. Des., 121, pp. 375-382.

[24] Gan, D., Dai, J. S., and Liao, Q., 2009, "Mobility Change in Two Types of Metamorphic Parallel Mechanisms," ASME J. Mech. Rob., 1, pp. 041007.

[25] Gan, D., Dai, J. S., Dias, J., and Seneviratne, L. D., 2013, "Unified Kinematics and Singularity Analysis of A Metamorphic Parallel Mechanism with Bifurcated Motion,” ASME J. Mech. Rob., 5(3), pp. 041104.

[26] Zhang, K., Dai, J.S., and Fang, Y.F., 2012, "Constraint analysis and bifurcated motion of the 3PUP parallel mechanism," Mech. Mach. Theory, 49, pp. 256-269.

[27] Zhang, K., Dai, J.S., and Fang, Y., 2010, "Topology and Constraint Analysis of Phase Change in the Metamorphic Chain and Its Evolved Mechanism,” ASME J. Mech. Des.,. 132, pp. 121001.

[28] Zhang, L., and Dai, J. S., 2009, "Reconfiguration of Spatial Mechanisms," ASME J. Mech. Rob., 1, pp. 011012.

[29] Kuo, C. H., Dai, J. S., and Yan, H. S., 2009, "Reconfiguration Principles and Strategies for Reconfigurable Mechanisms," ASME/IFToMM International Conference on Reconfigurable Mechanisms and Robots (ReMAR 2009), London, United Kingdom, 22-24 June 2009, pp. 1-7.

[30] Sun, T., Song, Y., Li, Y., and Zhang, J., 2010, "Workspace Decomposition Based Dimensional Synthesis of a Novel Hybrid Reconfigurable Robot,” ASME J. Mech. Rob., 2, pp. 031009.

[31] Fanghella, P., and Galletti, C., 1995, "Metric Relations and Displacement Groups in Mechanism and Robot Kinematics," ASME J. Mech. Des., 117, pp. 470-478.

[32] Hervé, J. M., 1999, "The Lie group of rigid body displacements, a fundamental tool for mechanism design," Mech. Mach. Theory, 34, pp. 719-730. 


\section{Listing of figure and table captions}

\section{Figures}

Fig. 1. Two motion modes of a single closed loop kinematotropic mechanism with $1 R \leftrightarrow 1 T$ form

Fig. 2. Basic characteristic structure of a serial kinematic chains with variable constraint relations

Fig. 3. Basic constraint forms of kinematotropic mechanisms

Fig. 4. Logical relations between the design steps of kinematotropic mechanisms

Fig. 5. Two kinematotropic mechanisms with motion transformation $3 R 1 T_{z}(3 D 4 F) \leftrightarrow 1 R_{z} 3 T(3 D 4 F)$

Fig. 6. Motion modes of the kinematotropic mechanism $3 R 1 T_{z}(3 D 4 F) \leftrightarrow 1 R_{z} 3 T(3 D 4 F)$ in the selftransforming form

Fig. 7. Motion modes of the kinematotropic mechanism $3 R 1 T_{z}(3 D 4 F) \leftrightarrow 1 R_{z} 3 T(3 D 4 F)$ in the intertransforming form

Fig. 8. Two kinematotropic mechanisms with motion transformation $1 R_{y} 1 T_{z}(2 D 2 F) \leftrightarrow 1 R_{z} 2 T_{x y}(3 D 3 F)$

Fig. 9. Motion modes of the kinematotropic mechanism $1 R_{y} 1 T_{z}(2 D 2 F) \leftrightarrow 1 R_{z} 2 T_{x y}(3 D 3 F)$ in the selftransforming form

Fig. 10. Motion modes of the kinematotropic mechanism $1 R_{y} 1 T_{z}(2 D 2 F) \leftrightarrow 1 R_{z} 2 T_{x y}(3 D 3 F)$ in the intertransforming form

\section{Tables}

Table 1. Basic spatial motions

Table 2. Basic serial chains

Table 3. Basic parallel mechanisms

Table 4. Basic serial kinematic chains with variable constraint relations 